\title{
Parametric pseudodifferential operators with point-singularity in the covariable
}

\author{
Jörg Seiler ${ }^{1}$ \\ Received: 23 December 2020 / Accepted: 23 December 2021 / Published online: 19 January 2022 \\ (c) The Author(s) 2022
}

\begin{abstract}
Starting out from a new description of a class of parameter-dependent pseudodifferential operators with finite regularity number due to G. Grubb, we introduce a calculus of parameterdependent, poly-homogeneous symbols whose homogeneous components have a particular type of point-singularity in the covariable-parameter space. Such symbols admit intrinsically a second kind of expansion which is closely related to the expansion in the Grubb-Seeley calculus and permits to recover the resolvent-trace expansion for elliptic pseudodifferential operators originally proved by Grubb-Seeley. Another application is the invertibility of parameter-dependent operators of Toeplitz type, i.e., operators acting in subspaces determined by zero-order pseudodifferential idempotents.
\end{abstract}

Keywords Pseudodifferential operators · Parameter-ellipticity · Resolvent · Trace expansion · Operators of Toeplitz type

Mathematics Subject Classification 58J40 · 47L80 • 47A10

\section{Introduction}

In the present paper, we develop a calculus of parameter-dependent pseudodifferential operators $(\psi \mathrm{do})$, both for operators in Euclidean space $\mathbb{R}^{n}$ and for operators on sections of vector-bundles over closed Riemannian manifolds, which is closely related to Grubb's calculus of operators with finite regularity number [3] (for a recent application to fractional heat equations see [5]) and to the Grubb-Seeley calculus introduced in [6]. The calculus allows to obtain the classical resolvent-trace expansion for elliptic $\psi$ do due to [6] and a systematic treatment of $\psi$ do of Toeplitz type in the sense of [14,15].

At the base of our calculus lies a "geometric" characterization of the above-mentioned regularity number: consider a parameter-dependent $\psi$ do $a(D ; \mu)$ with symbol $a(\xi ; \mu)$ depending, for simplicity, only on the covariable $\xi \in \mathbb{R}^{n}$ and the parameter $\mu \in \overline{\mathbb{R}}_{+}$. The symbol $a$ belongs to the parameter-dependent poly-homogeneous Hörmander class $S^{d}$ if it admits an asymptotic expansion

$\triangle$ Jörg Seiler

joerg.seiler@unito.it

1 Dipartimento di Matematica, Università di Torino, Turin, Italy 


$$
a(\xi ; \mu) \sim \sum_{j=0}^{+\infty} a_{j}(\xi ; \mu)
$$

with symbols $a_{j} \in S_{h o m}^{d-j}$ that are positively homogeneous in $(\xi, \mu)$ of degree $d-j$. If $\mathbb{S}_{+}^{n}=\left\{\left.(\xi, \mu)|| \xi\right|^{2}+\mu^{2}=1\right\}$ denotes the unit semi-sphere, then

$$
a_{j}(\xi ; \mu)=|(\xi, \mu)|^{d-j} \widehat{a}_{j}\left(\frac{(\xi, \mu)}{|(\xi, \mu)|}\right), \quad \widehat{a}_{j} \in \mathscr{C}^{\infty}\left(\mathbb{S}_{+}^{n}\right) .
$$

The operator is parameter-elliptic if the homogeneous principal symbol $a_{0}$ never vanishes, i.e., $\widehat{a}_{0}$ does never vanish on the unit semi-sphere. In this case there exists a parametrix $b(\xi ; \mu) \in S^{-d}$ such that $b(D ; \mu)$ is the inverse of $a(D ; \mu)$ for large $\mu$.

In Sect. 4 we show that $a(D ; \mu)$ is an operator of order $d$ and with regularity number $v \in \mathbb{R}$ in the sense of [3] if $a$ admits a decomposition $a=\widetilde{a}+p$ with $p \in S^{d}$ and where $\widetilde{a}$ admits an expansion of form (1.1), with components satisfying (1.2) but with singular functions $\widehat{a}_{j}$ : introducing polar coordinates $(r, \phi)$ on $\mathbb{S}_{+}^{n}$, centered in the "north-pole" $(\xi, \mu)=(0,1)$, they belong to the weighted space $r^{\nu-j} \mathscr{C}_{B}^{\infty}\left(\widehat{\mathbb{S}}_{+}^{n}\right)$, where $\widehat{\mathbb{S}}_{+}^{n}=\mathbb{S}_{+}^{n} \backslash\{(0,1)\}$ and $\mathscr{C}_{B}^{\infty}$ means smooth functions which remain bounded on $\widehat{\mathbb{S}}_{+}^{n}$ after arbitrary applications of totally characteristic derivatives $r \partial_{r}$ and usual derivatives in $\phi$.

This observation leads us to consider symbols $a=\widetilde{a}+p$ with $p \in S^{d}$ but where the homogeneous components of $\tilde{a}$ originate from the weighted spaces $r^{\nu-j} \mathscr{C}_{T}^{\infty}\left(\widehat{\mathbb{S}}_{+}^{n}\right), v \in \mathbb{Z}$, where $\mathscr{C}_{T}^{\infty}\left(\widehat{\mathbb{S}}_{+}^{n}\right)$ is the space of all functions on $\widehat{\mathbb{S}}_{+}^{n}$ that, in coordinates $(r, \phi)$, extend smoothly up to and including $r=0$ (the subscript $T$ stands for Taylor expansion). Symbols of this kind do not only have an expansion (1.1) but intrinsically a further expansion of the form

$$
a(\xi ; \mu) \sim \sum_{j=0}^{+\infty} a_{[v+j]}^{\infty}(\xi)[\xi, \mu]^{d-v-j}, \quad a_{[v+j]}^{\infty}(\xi) \in S^{\nu+j}\left(\mathbb{R}^{n}\right),
$$

where $[\xi, \mu]$ denotes a smooth function that coincides with the usual modulus away from the origin and $S^{m}\left(\mathbb{R}^{n}\right)$ is the standard poly-homogeneous Hörmander class of order $m$ without parameter. See Sect. 5 for details. Evidently, expansion (1.3) resembles the one employed by Grubb-Seeley in [6]. While Grubb-Seeley's expansion is in powers of $\mu$ and has its origin in a meromorphic (at infinity) dependence on the parameter $\mu,(1.3)$ directly originates from the Taylor expansion of the homogeneous components and makes no use of a holomorphic dependence on the parameter. However, expanding $[\xi, \mu]^{m}$ in powers of $\mu$ allows us to obtain a Grubb-Seeley expansion and ultimately we can recover the resolvent-trace expansion of $\psi$ do shown in [6]. This is discussed in detail in Sects. 6 and 7.6.

Ellipticity in our class is most simple for a positive regularity number $v>0$. In this case, the homogeneous principal symbol extends by continuity to the north-pole, and its non-vanishing yields the existence of a parametrix which is the inverse of $a(D ; \mu)$ for large values of the parameter $\mu$. For $v=0$, ellipticity is more involved and two additional symbolic levels come into play:

(a) the principal angular symbol which originates from the leading term of the Taylor expansion of the homogeneous principal symbol,

(b) the principal limit-symbol, i.e., the symbol $a_{[0]}^{\infty}$ from (1.3).

Non-vanishing of the homogeneous principal symbol, of the principal angular symbol, and invertibility of the operator $a_{[0]}^{\infty}(D)$ guarantee the existence of a parametrix in the class which is the inverse for large values of $\mu$. Concerning $(a)$, our calculus appears to be related with Savin-Sternin [8] where a similar structure occurs. 
We show that our calculus of operators on $\mathbb{R}^{n}$ is invariant under changes of coordinates, see Sect. 7.1. Thus, we can define corresponding classes of $\psi$ do on closed manifolds $M$, acting on sections of finite-dimensional vector-bundles. While the homogeneous principal symbol and the principal angular symbol have a global meaning as bundle morphisms on $\left(T^{*} M \times \overline{\mathbb{R}}_{+}\right) \backslash 0$ and $T^{*} M \backslash 0$, respectively, expansion (1.3) is shown to have a global analog too, namely

$$
A(\mu) \sim \sum_{j=0}^{+\infty} A_{[v+j]}^{\infty} \Lambda^{d-v-j}(\mu), \quad A_{[v+j]}^{\infty} \in L^{v+j}\left(M ; E_{0}, E_{1}\right),
$$

where $\Lambda^{\alpha}(\mu) \in L^{\alpha}\left(E_{0}, E_{0}\right), \alpha \in \mathbb{R}$, denote elliptic elements in Hörmander's class with (scalar) homogeneous principal symbol $\left(|\xi|_{x}^{2}+\mu^{2}\right)^{\alpha / 2}$, where $|\cdot|$ refers to some fixed Riemannian metric on $M$. This is shown in Sect. 7.2. The so-called limit-operator $A_{[\nu]}^{\infty}$ takes the place of the above used limit-symbol. In Sect. 7.7 we discuss an application to parameterdependent $\psi$ do of Toeplitz type, here on closed manifolds; originally the concept of Toeplitz type operators emerged in the study of boundary value problems with Atiyah-Patodi-Singer type boundary conditions, see $[12,13]$.

In the present paper, we limit ourselves to $\psi$ do on $\mathbb{R}^{n}$ or closed manifolds. However, it is a natural question whether the established calculus allows to build up a corresponding calculus for boundary value problems, in the spirit of [3,4] and [9], leading to a parameterdependent version of the classical Boutet de Monvel algebra [1]. Similarly, one could address the analogous question for manifolds with singularities (conical singularities, in the simplest case), following and extending the approach of Schulze $[10,11]$. We plan to address these questions in future work.

Hoping to help the reader in reading this paper, we finish this introduction by listing the most important spaces of pseudodifferential symbols used in the sequel:

$$
\begin{aligned}
S_{1,0}^{d}\left(\mathbb{R}^{n}\right), S^{d}\left(\mathbb{R}^{n}\right): & \text { Section } 2.2 \\
S_{1,0}^{d, v}, S^{d, v}, S_{\text {hom }}^{d, v}: & \text { Definitions 3.1, 3.3, and 3.4 } \\
S_{1,0}^{d}, S^{d}, S_{\text {hom }}^{d}: & \text { Definitions 3.7, 3.9, and 3.10 } \\
\widetilde{S}_{1,0}^{d, \nu}, \widetilde{S}^{d, v}, \widetilde{S}_{\text {hom }}^{d, v}: & \text { Definitions 3.12, 3.15, and 3.14 } \\
\widetilde{\mathbf{S}}_{1,0}^{d, v}, \widetilde{\mathbf{S}}^{d, v}, \widetilde{\mathbf{S}}_{\text {hom }}^{d, v}: & \text { Definitions 5.1, 5.14 and 5.12 } \\
\mathbf{S}^{d, v}, \mathbf{S}_{\text {hom }}^{d, v}: & \text { Definition 5.18 }
\end{aligned}
$$

\section{Notations, symbols, and Leibniz product}

\subsection{Basic notations}

Let $\langle y\rangle=\left(1+|y|^{2}\right)^{1 / 2}$ for $y \in \mathbb{R}^{m}$ with arbitrary $m$. Let $y \mapsto[y]: \mathbb{R}^{m} \rightarrow \mathbb{R}$ denote a smooth, strictly positive function that coincides with the modulus $|y|$ outside the unit-ball. If $y=(\xi, \mu)$, we write shortly $|\xi, \mu|:=|(\xi, \mu)|,\langle\xi, \mu\rangle=\langle(\xi, \mu)\rangle$, and $[\xi, \mu]:=[(\xi, \mu)]$.

A zero-excision function on $\mathbb{R}^{m}$ is a smooth function $\chi(y)$ that vanishes in a neighborhood of the origin and such that $(1-\chi)(y)$ has compact support.

If $f, g: \Omega \rightarrow \mathbb{R}$ are two functions on some set $\Omega$ we shall write $f \lesssim g$ or $f(\omega) \lesssim g(\omega)$ if there exists a constant $C \geq 0$ such that $f(\omega) \leq C g(\omega)$ for every $\omega \in \Omega$. 
Let $f(\omega, y)$ be defined on a set of the form $\Omega \times\left(\mathbb{R}^{m} \backslash\{0\}\right)$. With slight abuse of language, we shall call $f$ homogeneous of degree $d$ in $y$ if

$$
f(\omega, t y)=t^{d} f(\omega, y) \quad \forall(\omega, y) \quad \forall t>0 ;
$$

it would be correct to use the terminology positively homogeneous, but for brevity we shall not do so. Suppose $y=(u, v)$ with $u \in \mathbb{R}^{k}$ and $v \in \mathbb{R}^{m-k}$ ( $k$ may be equal to $m$, i.e., $y=u$ ). We shall say that $f$ is homogeneous of degree $d$ in $(u, v)$ for large $u$ if there exists a constant $R \geq 0$ (frequently assumed to be equal to 1 ) such that

$$
f(\omega, t u, t v)=t^{d} f(\omega, u, v) \quad \forall(\omega, v) \quad \forall|u| \geq R \quad \forall t \geq 1 .
$$

\subsection{Hörmander's class}

The uniform Hörmander class $S_{1,0}^{d}\left(\mathbb{R}^{n}\right)$ consists of those symbols $a(x, \xi): \mathbb{R}^{n} \times \mathbb{R}^{n} \rightarrow \mathbb{C}$ satisfying the uniform estimates

$$
\left|D_{\xi}^{\alpha} D_{x}^{\beta} a(x, \xi)\right| \lesssim\langle\xi\rangle^{d-|\alpha|}
$$

for every multi-indices $\alpha, \beta \in \mathbb{N}_{0}^{n}$. This is a Fréchet space with the system of norms

$$
\|a\|_{j}=\max _{|\alpha|+|\beta| \leq j} \sup _{x, \xi}\left|D_{\xi}^{\alpha} D_{x}^{\beta} a(x, \xi)\right|\langle\xi\rangle^{|\alpha|-d}, \quad j \in \mathbb{N}_{0} .
$$

Let us denote by $S_{\text {hom }}^{d}\left(\mathbb{R}^{n}\right)$ the space of all smooth functions $a(x, \xi)$ defined on $\mathbb{R}^{n} \times\left(\mathbb{R}^{n} \backslash\{0\}\right)$ which are homogeneous of degree $d$ in $\xi$ and satisfy

$$
\left|D_{\xi}^{\alpha} D_{x}^{\beta} a(x, \xi)\right| \lesssim|\xi|^{d-|\alpha|} .
$$

A symbol $a(x, \xi) \in S_{1,0}^{d}\left(\mathbb{R}^{n}\right)$ is called poly-homogeneous if there exist functions $a_{\ell}(x, \xi) \in$ $S_{\text {hom }}^{d-\ell}\left(\mathbb{R}^{n}\right)$ such that

$$
r_{a, N}(x, \xi):=a(x, \xi)-\chi(\xi) \sum_{\ell=0}^{N-1} a_{\ell}(x, \xi) \in S_{1,0}^{d-N}\left(\mathbb{R}^{n}\right)
$$

for every $N$, where $\chi(\xi)$ is an arbitrary fixed zero-excision function (note that $r_{a, 0}=a$ ). Denote by $S^{d}\left(\mathbb{R}^{n}\right)$ the space of all such symbols. It is a Fréchet space with the system of norms $\|a\|_{j, N}:=\left\|r_{a, N}\right\|_{j}, j, N \in \mathbb{N}_{0}$, and

$$
\|a\|_{j, \ell}^{\prime}=\max _{|\alpha|+|\beta| \leq j} \sup _{x,|\xi|=1}\left|D_{\xi}^{\alpha} D_{x}^{\beta} a_{\ell}(x, \xi)\right|, \quad j, \ell \in \mathbb{N}_{0}
$$

The $\psi$ do associated with $a(x, \xi)$, denoted by $a(x, D)$, is

$$
[a(x, D) u](x)=\int e^{i x \xi} a(x, \xi) \widehat{u}(\xi) d \xi, \quad x \in \mathbb{R},
$$

acting on the Schwartz space $\mathscr{S}\left(\mathbb{R}^{n}\right)$ of rapidly decreasing functions; here, $d \xi=(2 \pi)^{-n} d \xi$. The composition of operators $a_{0}(x, D)$ and $a_{1}(x, D)$ corresponds to the so-called Leibniz product of symbols,

$$
\left(a_{1} \# a_{0}\right)(x, \xi)=\iint e^{i y \eta} a_{1}(x, \xi+\eta) a_{0}(x+y, \xi) d y d \eta .
$$


(integration in the sense of oscillatory integrals), cf. for example [7]. If the $a_{j}$ have order $d_{j}$, then $a_{1} \# a_{0}$ has order $d_{0}+d_{1}$. The adjoint symbol

$$
a^{(*)}(x, \xi)=\iint e^{-i y \eta} \overline{a(x+y, \xi+\eta ; \mu)} d y d \eta
$$

gives the formal adjoint operator of $a(x, D)$, i.e.,

$$
(a(x, D) u, v)_{L^{2}}=\left(u, a^{(*)}(x, D) v\right)_{L^{2}}, \quad u, v \in \mathscr{S}\left(\mathbb{R}^{n}\right) .
$$

If $a(x, \xi ; \mu)$ is a symbol that depends on an additional parameter $\mu$, we shall write $a(x, D ; \mu)$, Leibniz product and adjoint are applied point-wise in $\mu$.

Throughout the paper we consider a parameter $\mu \in \overline{\mathbb{R}}_{+}:=[0,+\infty)$.

\section{Symbols with finite regularity number}

\subsection{Grubb's calculus}

We briefly review a pseudodifferential calculus introduced by Grubb. For further details, we refer the reader to Chapter 2.1 of [3].

Definition 3.1 By $S_{1,0}^{d, v}$ with $d, v \in \mathbb{R}$ (called order and regularity number, respectively) denote the space of all symbols $a(x, \xi ; \mu)$ satisfying

$$
\left|D_{\xi}^{\alpha} D_{x}^{\beta} D_{\mu}^{j} a(x, \xi ; \mu)\right| \lesssim\langle\xi\rangle^{\nu-|\alpha|}\langle\xi, \mu\rangle^{d-\nu-j}+\langle\xi, \mu\rangle^{d-|\alpha|-j} .
$$

The space of smoothing or regularizing symbols, defined as

$$
S^{d-\infty, v-\infty}=\bigcap_{N \geq 0} S_{1,0}^{d-N, v-N},
$$

consists of those symbols satisfying, for every $N$ and all orders of derivatives,

$$
\left|D_{\xi}^{\alpha} D_{x}^{\beta} D_{\mu}^{j} a(x, \xi ; \mu)\right| \lesssim\langle\xi\rangle^{-N}\langle\mu\rangle^{d-\nu-j}
$$

Proposition 3.2 The Leibniz product induces maps

$$
S_{1,0}^{d_{1}, v_{1}} \times S_{1,0}^{d_{0}, v_{0}} \longrightarrow S_{1,0}^{d_{0}+d_{1}, v}, \quad v=\min \left(v_{0}, v_{1}, v_{0}+v_{1}\right) .
$$

Asymptotic summations can be performed within the class, in the following sense: Given a sequence of symbols $a_{\ell} \in S_{1,0}^{d-\ell, v-\ell}$, there exists an $a \in S_{1,0}^{d, v}$ such that

$$
a(x, \xi ; \mu)-\sum_{\ell=0}^{N-1} a_{\ell}(x, \xi ; \mu) \in S_{1,0}^{d-N, \nu-N}
$$

for every $N$; $a$ is uniquely determined modulo $S^{d-\infty, \nu-\infty}$.

Definition 3.3 A symbol $a \in S_{1,0}^{d, v}$ is called poly-homogeneous if it satisfies (3.4) with $a_{\ell} \in$ $S_{1,0}^{d-\ell, \nu-\ell}$ that are homogeneous of degree $d-\ell$ in $(\xi, \mu)$ for $|\xi| \geq 1$. The space of these symbols is denoted by $S^{d, v}$. 
If $a \in S^{d, v}$, its homogeneous principal symbol is defined as

$$
a^{h}(x, \xi ; \mu):=|\xi|^{d} a_{0}\left(x, \frac{\xi}{|\xi|} ; \frac{\mu}{|\xi|}\right)=\lim _{t \rightarrow+\infty} t^{-d} a(x, t \xi ; t \mu), \quad \xi \neq 0 .
$$

It satisfies

$$
\left|D_{\xi}^{\alpha} D_{x}^{\beta} D_{\mu}^{j} a^{h}(x, \xi, \mu)\right| \lesssim|\xi|^{\nu-|\alpha|}|\xi, \mu|^{d-\nu-j}+|\xi, \mu|^{d-|\alpha|-j}
$$

Definition 3.4 $S_{\text {hom }}^{d, v}$ denotes the space of all smooth functions $a(x, \xi ; \mu)$ defined for $\xi \neq$ 0 , which are homogeneous of degree $d$ in $(\xi, \mu)$ and satisfy (3.5) for arbitrary orders of derivatives.

If $a \in S_{\text {hom }}^{d, v}$ and $v>0$, then $a$ extends by continuity to a function defined for all $x$ and $(\xi, \mu) \neq 0$; the larger $v$ is, the more regular (i.e., differentiable) is this extension. This is the justification for the terminology "regularity number." In this case we shall identify $a$ with its extension.

Definition 3.5 A symbol $a(x, \xi ; \mu) \in S^{d, v}, v>0$, is called elliptic if $a^{h}(x, \xi ; \mu) \neq 0$ for all $x$ and all $(\xi, \mu) \neq 0$ and $\left|a^{h}(x, \xi, \mu)^{-1}\right| \lesssim|\xi, \mu|^{-d}$.

Note that if $a^{h}(x, \xi ; \mu)$ is constant in $x$ for large $x$, it suffices to require the pointwise invertibility of $a^{h}(x, \xi, \mu)$

Theorem 3.6 Let $v>0$ and $a \in S^{d, v}$. Then a is elliptic if and only if there exists $a b \in S^{-d, v}$ such that $a \# b-1, b \# a-1 \in S^{0-\infty, \nu-\infty}$.

Note that if $r \in S^{0-\infty, v-\infty}$ with $v>0$, then $r(\mu) \stackrel{\mu \rightarrow+\infty}{\longrightarrow} 0$ in $S^{-\infty}\left(\mathbb{R}^{n}\right)$. In particular, if $a$ is elliptic then $a(x, D ; \mu)$ is invertible for large $\mu$.

(3.1) and (3.5) suggest to introduce two subspaces of $S_{1,0}^{d, v}$ and $S_{h o m}^{d, v}$, respectively, with estimates corresponding to the first and second term on the right-hand side, respectively. These will be discussed in the next two subsections.

\subsection{Strong parameter-dependence (symbols of infinite regularity)}

In this section, we consider the space $S_{1,0}^{d}=\bigcap_{N \geq 0} S_{1,0}^{d, N}$ and the poly-homogeneous subclass. For clarity we prefer to present it in an independent way.

Definition $3.7 S_{1,0}^{d}$ consists of all symbols $a(x, \xi ; \mu)$ satisfying, for all orders of derivatives,

$$
\left|D_{\xi}^{\alpha} D_{x}^{\beta} D_{\mu}^{j} a(x, \xi ; \mu)\right| \lesssim\langle\xi, \mu\rangle^{d-|\alpha|-j} .
$$

We shall call such symbols also strongly parameter-dependent, since differentiation with respect to $\xi$ or $\mu$ improves the decay in $(\xi, \mu)$.

The space of regularizing symbols $S^{-\infty}=\cap_{d \in \mathbb{R}} S^{d}$ consists of those symbols which are rapidly decreasing in $(\xi, \mu)$ and $\mathscr{C}_{b}^{\infty}$ in $x$.

Proposition 3.8 The Leibniz product induces maps $S_{1,0}^{d_{1}} \times S_{1,0}^{d_{0}} \rightarrow S_{1,0}^{d_{0}+d_{1}}$.

Definition 3.9 $S_{\text {hom }}^{d}$ consist of all symbols $a(x, \xi ; \mu)$ defined for $(\xi, \mu) \neq 0$ which are homogeneous of degree $d$ in $(\xi, \mu)$ and satisfy, for every order of derivatives,

$$
\left|D_{\xi}^{\alpha} D_{x}^{\beta} D_{\mu}^{j} a(x, \xi ; \mu)\right| \lesssim|\xi, \mu|^{d-|\alpha|-j}
$$


Definition 3.10 A symbol $a \in S_{1,0}^{d}$ is called poly-homogeneous if there exists a sequence of homogeneous symbols $a_{\ell} \in S_{\text {hom }}^{d-\ell}$ such that, for every $N$,

$$
a(x, \xi ; \mu)-\sum_{\ell=0}^{N-1} \chi(\xi, \mu) a_{\ell}(x, \xi ; \mu) \in S_{1,0}^{d-N},
$$

where $\chi(\xi, \mu)$ is an arbitrary zero-excision function. The space of such symbols will be denoted by $S^{d}$.

We call $a_{0}$ the homogeneous principal symbol of $a \in S^{d}$, and

$$
a_{0}(x, \xi ; \mu)=\lim _{t \rightarrow+\infty} t^{-d} a(x, t \xi ; t \mu), \quad(\xi, \mu) \neq 0 .
$$

Ellipticity of $a$ is defined as in Definition 3.5 and the obvious analog of Theorem 3.6 is valid.

Remark 3.11 In the literature, the space $S^{d}$ is frequently denoted by $S_{\mathrm{cl}}^{d}$ and the symbols are called classical rather than poly-homogeneous.

\subsection{Weakly parameter-dependent symbols}

Let us describe the second natural subspace of $S_{1,0}^{d, v}$.

Definition 3.12 Let $\widetilde{S}_{1,0}^{d, v}$ denote the space of all symbols $a(x, \xi ; \mu)$ which satisfy, for every order of derivatives,

$$
\left|D_{\xi}^{\alpha} D_{x}^{\beta} D_{\mu}^{j} a(x, \xi ; \mu)\right| \lesssim\langle\xi\rangle^{\nu-|\alpha|}\langle\xi, \mu\rangle^{d-\nu-j} .
$$

Note that $\widetilde{S}_{1,0}^{d, v}=S_{1,0}^{d, v}$ whenever $v \leq 0$. In particular, $\widetilde{S}_{1,0}^{d-\infty, v-\infty}=S_{1,0}^{d-\infty, v-\infty}$

Proposition 3.13 The Leibniz product induces maps

$$
\widetilde{S}_{1,0}^{d_{1}, v_{1}} \times \widetilde{S}_{1,0}^{d_{0}, v_{0}} \rightarrow \widetilde{S}_{1,0}^{d_{0}+d_{1}, v_{0}+v_{1}} .
$$

Definition 3.14 Let $\widetilde{S}_{\text {hom }}^{d, v}$ denote the space of all functions $a(x, \xi ; \mu)$ which are defined for $\xi \neq 0$, are homogeneous in $(\xi, \mu)$ of degree $d$ and satisfy, for every order of derivatives,

$$
\left|D_{\xi}^{\alpha} D_{x}^{\beta} D_{\mu}^{j} a(x, \xi ; \mu)\right| \lesssim|\xi|^{\nu-|\alpha|}|\xi, \mu|^{d-\nu-j} .
$$

Definition 3.15 A symbol $a \in \widetilde{S}_{1,0}^{d, v}$ is called poly-homogeneous if there exists a sequence of homogeneous symbols $a_{\ell} \in \widetilde{S}_{h o m}^{d-\ell, \nu-\ell}$ such that, for every $N$,

$$
a(x, \xi ; \mu)-\sum_{\ell=0}^{N-1} \chi(\xi) a_{\ell}(x, \xi ; \mu) \in \widetilde{S}_{1,0}^{d-N, \nu-N},
$$

where $\chi(\xi)$ is an arbitrary zero-excision function. The space of such symbols will be denoted by $\widetilde{S}^{d, v}$.

Again, $a_{0}$ is called the homogeneous principal symbol of $a \in \widetilde{S}^{d, v}$, and

$$
a_{0}(x, \xi ; \mu)=\lim _{t \rightarrow+\infty} t^{-d} a(x, t \xi ; t \mu), \quad \xi \neq 0 .
$$


Definition 3.16 A symbol $a(x, \xi ; \mu) \in \widetilde{S}^{d, v}$ is called elliptic if $a_{0}(x, \xi ; \mu) \neq 0$ for all $x, \mu$ and all $\xi \neq 0$, and $\left|a_{0}(x, \xi, \mu)^{-1}\right| \lesssim|\xi|^{-v}|\xi, \mu|^{-d+v}$.

Theorem 3.17 A symbol $a \in \widetilde{S}^{d, v}$ is elliptic if and only if there exists $a b \in \widetilde{S}^{-d,-v}$ such that $a \# b-1, b \# a-1 \in \widetilde{S}^{0-\infty, 0-\infty}$.

Note that ellipticity of $a \in \widetilde{S}^{d, v}$ is not equivalent to the point-wise invertibility of the homogeneous principal symbol $a_{0}$ on its domain, even not in case of independence of the $x$-variable (see Theorem 4.4 and the subsequent comment). Moreover, a remainder $r \in$ $\widetilde{S}^{0-\infty, 0-\infty}$ is, in general, only bounded but not decaying as $\mu \rightarrow+\infty$. Therefore, $a(x, D ; \mu)$ need not be invertible for large $\mu$.

\section{Regularity number and weighted spaces}

In any of the so far introduced symbol spaces, the involved variable $x$ enters as a $\mathscr{C}_{b}^{\infty}$-variable, while the spaces differ by the structures in the variables $(\xi, \mu)$. For this reason, and also to keep notation more lean, in this section we ignore the $x$-dependence and focus on symbols depending only on $(\xi, \mu)$.

Let us denote by $\mathbb{S}_{+}^{n}$ the unit semi-sphere,

$$
\mathbb{S}_{+}^{n}:=\left\{(\xi, \mu) \in \mathbb{R}^{n} \times\left.\overline{\mathbb{R}}_{+}|| \xi\right|^{2}+\mu^{2}=1\right\} .
$$

Every homogeneous symbol $a \in S_{\text {hom }}^{d}$ is of the form

$$
a(\xi ; \mu)=|\xi, \mu|^{d} \widehat{a}\left(\frac{(\xi, \mu)}{|\xi, \mu|}\right), \quad \widehat{a}=\left.a\right|_{\mathbb{S}_{+}^{n}} \in \mathscr{C}^{\infty}\left(\mathbb{S}_{+}^{n}\right)
$$

and the map $a \mapsto \widehat{a}$ establishes an isomorphism between $S_{h o m}^{d}$ and $\mathscr{C}^{\infty}\left(\mathbb{S}_{+}^{n}\right)$. A symbol $a \in \widetilde{S}_{h o m}^{d, v}$ is defined for $\xi \neq 0$ only, hence its restriction is defined only on the punctured unit semi-sphere

$$
\widehat{\mathbb{S}}_{+}^{n}:=\mathbb{S}_{+}^{n} \backslash\{(0,1)\}=\left\{(\xi, \mu) \in \mathbb{S}_{+}^{n} \mid \xi \neq 0\right\}
$$

We shall now investigate, which subspace of $\mathscr{C}^{\infty}\left(\widehat{\mathbb{S}}_{+}^{n}\right)$ is in 1-1-correspondence with $\widetilde{S}_{\text {hom }}^{d, v}$. To this end, we shall identify $\widehat{\mathbb{S}}_{+}^{n}$ with $(0,1] \times \mathbb{S}^{n-1}$, using the (polar-)coordinates

$$
\xi=r \phi, \quad \mu=\sqrt{1-r^{2}}, \quad\left(0<r \leq 1, \quad \phi \in \mathbb{S}^{n-1}\right) .
$$

If $E$ is an arbitrary Fréchet space, we shall denote by $\mathscr{C}_{B}^{\infty}((0, \varepsilon), E)$ the space of all smooth bounded functions $u:(0, \varepsilon) \rightarrow E$ such that $\left(r \partial_{r}\right)^{\ell} u$ is bounded on $(0, \varepsilon)$ for every order of derivatives.

Definition 4.1 With $\gamma \in \mathbb{R}$ define

$$
\begin{aligned}
\mathscr{C}_{B}^{\infty}\left(\widehat{\mathbb{S}}_{+}^{n}\right) & :=\left\{a \in \mathscr{C}^{\infty}\left(\widehat{\mathbb{S}}_{+}^{n}\right) \mid a(r, \phi) \in \mathscr{C}_{B}^{\infty}\left((0, \varepsilon), \mathscr{C}^{\infty}\left(\mathbb{S}^{n-1}\right)\right) \text { for some } \varepsilon>0\right\}, \\
r^{\gamma} \mathscr{C}_{B}^{\infty}\left(\widehat{\mathbb{S}}_{+}^{n}\right) & :=\left\{a \in \mathscr{C}^{\infty}\left(\widehat{\mathbb{S}}_{+}^{n}\right) \mid r^{-\gamma} a \in \mathscr{C}_{B}^{\infty}\left(\widehat{\mathbb{S}}_{+}^{n}\right)\right\} .
\end{aligned}
$$

In other words, the index $\gamma$ indicates the rate of (non-)vanishing in the point $(\xi, \mu)=$ $(0,1)$; we shall also speak of spaces with weight $\gamma$. Note that $|\xi|=r$. 
Definition 4.2 Let $\widetilde{S}^{(d, \gamma)}$ denote the space of all functions $a(\xi ; \mu)$ defined for $\xi \neq 0$ of the form

$$
a(\xi ; \mu)=|\xi, \mu|^{d} \widehat{a}\left(\frac{(\xi, \mu)}{|\xi, \mu|}\right), \quad \widehat{a} \in r^{\gamma} \mathscr{C}_{B}^{\infty}\left(\widehat{\mathbb{S}}_{+}^{n}\right) .
$$

Let $a$ and $\widehat{a}$ be as in the previous definition. Identifying $\widehat{a}(\xi, \mu)$ with its local representation $\widehat{a}(r, \phi)$, we have the relations

$$
\widehat{a}(r, \phi)=a\left(r \phi ; \sqrt{1-r^{2}}\right), \quad a(\xi ; \mu)=|\xi, \mu|^{d} \widehat{a}\left(\frac{|\xi|}{|\xi, \mu|}, \frac{\xi}{|\xi|}\right) .
$$

In particular, the $d$-homogeneous extension of $\widehat{a}(r, \phi)=r^{v}$ is $a(\xi ; \mu)=|\xi|^{v}|\xi, \mu|^{d-v}$.

Lemma 4.3 Let $\widehat{\chi} \in \mathscr{C}^{\infty}\left(\mathbb{S}_{+}^{n}\right)$ vanish in a small neighborhood of $(\xi, \mu)=(0,1)$ and let $\chi(\xi, \mu)=\widehat{\chi}((\xi, \mu) /|\xi, \mu|) \in S_{\text {hom }}^{0}$ be its homogeneous extension of degree 0 . Then

$$
\chi S_{\text {hom }}^{d}=\chi S_{\text {hom }}^{d, v}=\chi \widetilde{S}^{(d, v)} .
$$

In fact, it suffices to observe that $\widehat{\chi}(\xi, \mu)$ is supported in a set of the form $\{(\xi, \mu) \mid 0 \leq$ $\mu \leq c|\xi|\}$ on which $|\xi| \leq|\xi, \mu| \lesssim|\xi|$.

The following theorem shows that, for weakly parameter-dependent homogeneous components, regularity number and weight are the same thing.

Theorem 4.4 $\widetilde{S}^{(d, v)}=\widetilde{S}_{\text {hom }}^{d, v}$ for every $d, v \in \mathbb{R}$. In particular, the map $\left.a \mapsto a\right|_{\widehat{\mathbb{S}}_{+}^{n}}$ establishes an isomorphism between $\widetilde{S}_{\text {hom }}^{d, v}$ and $r^{v} \mathscr{C}_{B}^{\infty}\left(\widehat{\mathbb{S}}_{+}^{n}\right)$.

Proof Let us first prove the inclusion " $\subseteq$." Let $a(\xi ; \mu)$ be as in Definition 4.2. By multiplication with $|\xi, \mu|^{-d}$, we may assume without loss of generality that $d=0$. In view of Lemma 4.3 we may assume that $\widehat{a}$ is supported in a small neighborhood of the point $(0,1)$. Hence, in representation (4.4) we may assume that $\widehat{a}(r, \phi) \in r^{\nu} \mathscr{C}_{B}^{\infty}\left((0,1), \mathscr{C}^{\infty}\left(\mathbb{S}^{n-1}\right)\right)$. We also may assume $v=0$, since the homogeneous extension of degree $d=0$ of $r^{\nu}$ is just $|\xi|^{v}|\xi, \mu|^{-v}$. Thus we can write

$$
a(\xi ; \mu)=a^{\prime}\left(\frac{|\xi|}{|\xi, \mu|}, \xi\right), \quad a^{\prime}(r, \xi):=\widehat{a}\left(r, \frac{\xi}{|\xi|}\right) \in \mathscr{C}_{B}^{\infty}\left((0,1), S_{\text {hom }}^{0}\left(\mathbb{R}^{n}\right)\right) .
$$

Therefore,

$$
\partial_{\mu} a(\xi ; \mu)=\left(\partial_{r} a^{\prime}\right)\left(\frac{|\xi|}{|\xi, \mu|}, \xi\right) \partial_{\mu} \frac{|\xi|}{|\xi, \mu|}=-\left(\left(r \partial_{r}\right) a^{\prime}\right)\left(\frac{|\xi|}{|\xi, \mu|}, \xi\right) \frac{\mu}{|\xi, \mu|^{2}}
$$

as well as

$$
\partial_{\xi_{j}} a(\xi ; \mu)=\left(\partial_{\xi_{j}} a^{\prime}\right)\left(\frac{|\xi|}{|\xi, \mu|}, \xi\right)-\left(\left(r \partial_{r}\right) a^{\prime}\right)\left(\frac{|\xi|}{|\xi, \mu|}, \xi\right)\left(\frac{\xi_{j}}{|\xi|^{2}}+\frac{\mu}{|\xi, \mu|^{2}}\right) .
$$

By induction, we thus find that $D_{\xi}^{\alpha} D_{\mu}^{j} a(\xi ; \mu)$ is a finite linear combination of terms of the form

$$
a_{m}^{\prime}\left(\frac{|\xi|}{|\xi, \mu|}, \xi\right) p_{j+\ell}(\xi, \mu), \quad \ell, m \in \mathbb{N}_{0}, \quad \ell+m=|\alpha|,
$$

with $a_{m}^{\prime}(r, \xi) \in \mathscr{C}_{B}^{\infty}\left((0,1), S_{h o m}^{-m}\left(\mathbb{R}^{n}\right)\right)$ and $p_{j+\ell} \in S_{h o m}^{-(j+\ell)}$. This yields

$$
\left|D_{\xi}^{\alpha} D_{\mu}^{j} a(\xi ; \mu)\right| \lesssim \sum_{\substack{\ell+m=|\alpha| \\ \ell, m \in \mathbb{N}_{0}}}|\xi|^{-m}|\xi, \mu|^{-j-\ell} \lesssim|\xi|^{-|\alpha|}|\xi, \mu|^{-j}
$$


Next we shall show the inclusion " $\supseteq$." Let $a \in \widetilde{S}_{\text {hom }}^{d, v}$ be given. It is enough to consider the case $d=v=0$, since $a \in \widetilde{S}_{h o m}^{d, v}$ if and only if $|\xi|^{-v}|\xi, \mu|^{v-d} a \in \widetilde{S}_{h o m}^{0,0}$ and $|\xi|^{-v}|\xi, \mu|^{v-d}=r^{-v}$ in polar-coordinates. Again, $a$ can be assumed do have support in a small conical neighborhood containing $(0,1)$. Thus

$$
\widehat{a}(r, \phi)=a\left(r \phi, \sqrt{1-r^{2}}\right)=a(\phi ; v(r)), \quad v(r)=\frac{\sqrt{1-r^{2}}}{r},
$$

vanishes for $r \geq \delta$ for some $\delta<1$. Extend $\widehat{a}$ from $(0,1) \times \mathbb{S}^{n-1}$ to $(0,1) \times\left(\mathbb{R}^{n} \backslash\{0\}\right)$ by

$$
\widehat{a}(r, \phi)=a\left(\frac{\phi}{|\phi|} ; v(r)\right), \quad 0 \neq \phi \in \mathbb{R}^{n} .
$$

Using that $r v^{\prime}(r) / v(r)=1 /\left(r^{2}-1\right)$, it is straightforward to see that $\left(r \partial_{r}\right)^{\ell} \partial_{\phi}^{\alpha} \widehat{a}(r, \phi)$ is a linear combination of terms of the form

$$
\left(\left(\mu \partial_{\mu}\right)^{j} \partial_{\xi}^{\beta} a\right)\left(\frac{\phi}{|\phi|}, v(r)\right) q(\phi) g(r), \quad j \leq \ell, \quad \beta \leq \alpha,
$$

where $q$ is smooth and homogeneous of degree $-|\alpha|$ in $\phi \neq 0$ and $g \in \mathscr{C}^{\infty}([0,1))$. Thus $\left(r \partial_{r}\right)^{\ell} \partial_{\phi}^{\alpha} \widehat{a}(r, \phi)$ is bounded for $r \in(0, \delta]$ and $\phi$ belonging to a small neighborhood of the unit-sphere $\mathbb{S}^{n}$. This shows the claim.

In particular, we see that $\widetilde{S}_{h o m}^{d, v}$ does not behave well under inversion: if $a \in r^{v} \mathscr{C}^{\infty}\left(\widehat{\mathbb{S}}_{+}^{n}\right)$ is point-wise invertible, the inverse will, in general, not belong to such a weighted space. To guarantee this, an additional control at the singularity of $a$ is needed. This will be addressed in the sequel.

Theorem 4.5 $S_{\text {hom }}^{d, v}=\widetilde{S}_{\text {hom }}^{d, v}+S_{\text {hom }}^{d}$ for every $d, v \in \mathbb{R}$.

Proof The first identity is true in case $v \leq 0$, since then $S_{\text {hom }}^{d} \subseteq S_{\text {hom }}^{d, v}=\widetilde{S}_{\text {hom }}^{d, v}$ by definition of the involved spaces.

It remains to consider $v>0$. The inclusion $\supseteq$ is clear. By multiplication with $|\xi, \mu|^{-d}$ we may assume without loss of generality that $d=0$.

Let $a \in S_{\text {hom }}^{0, v}$ be given. We use Theorem 4.4 and show that the restriction of $a$ to $\mathbb{S}_{+}^{n}$ is the sum of a smooth function and a function belonging to $r^{\nu} \mathscr{C}_{B}^{\infty}\left(\widehat{\mathbb{S}}_{+}^{n}\right)$. By Lemma 4.3 it suffices to find a decomposition for $(1-\chi) a$.

Let $N$ be the largest natural number with $N<v$. It can be shown (see Lemma 2.1.10 and Proposition 2.1.11 in [3]) that $a$ extends as an $N$-times continuously differentiable function to $\mathbb{R}^{n} \times \overline{\mathbb{R}}_{+} \backslash\{(0,0)\}$ and if $p_{N}(\xi ; \mu)$ denotes the Taylor-polynomial of $a$ in $\xi$ around $\xi=0$, then $p_{N}$ is smooth in $\mu>0$ and

$$
r_{N}(\xi ; \mu):=a(\xi ; \mu)-p_{N}(\xi ; \mu)=\sum_{|\alpha|=N+1} \frac{N+1}{\alpha !} \xi^{\alpha} \int_{0}^{1}(1-t)^{N}\left(\partial_{\xi}^{\alpha} a\right)(t \xi ; \mu) d t .
$$

Since $(1-\chi) p_{N}$ is smooth on $\mathbb{S}_{+}^{n}$, it remains to verify that the restriction of $(1-\chi) r_{N}$ belongs to $r^{\nu} \mathscr{C}_{B}^{\infty}\left(\widehat{\mathbb{S}}_{+}^{n}\right)$. To this end let

$$
r_{\alpha}(\xi, \mu)=\xi^{\alpha} \int_{0}^{1}(1-t)^{N} \partial_{\xi}^{\alpha} a(t \xi ; \mu) d t, \quad|\alpha|=N+1 .
$$

Then, in polar-coordinates,

$$
\widehat{r}_{\alpha}(r, \phi)=\phi^{\alpha} \int_{0}^{1}(1-t)^{N}\left(\partial_{\xi}^{\alpha} a\right)(t \phi ; v(r)) d t, \quad v(r)=\frac{\sqrt{1-r^{2}}}{r} .
$$


It suffices to show that $\widehat{r}_{\alpha} \in r^{\nu} \mathscr{C}_{B}^{\infty}\left((0, \varepsilon), \mathscr{C}^{\infty}\left(\mathbb{S}^{n-1}\right)\right)$ for some $\varepsilon>0$. We have

$$
\left|\widehat{r}_{\alpha}(r, \phi)\right| \leq \int_{0}^{1}|t \phi|^{\nu-|\alpha|}|t \phi, v(r)|^{-v} d t=r^{v} \int_{0}^{1} t^{-1+(v-N)}\left|t r \phi, \sqrt{1-r^{2}}\right|^{-v} d t .
$$

Since $\left|\operatorname{tr} \phi, \sqrt{1-r^{2}}\right|^{-v} \lesssim 1$ for $r \leq \delta$. we find that $r^{-v}\left|\widehat{r}_{\alpha}(r, \phi)\right|$ is bounded. Derivatives of $\widehat{r}_{\alpha}$ are treated similarly, proceeding as in the proof of Theorem 4.4.

This decomposition also shows how to associate with a symbol $a \in S_{\text {hom }}^{d, v}$ a symbol $p \in S^{d, v}$ with homogeneous principal symbol equal to $a$. In fact, writing $a=\widetilde{a}+a_{\text {smooth }}$ with $\widetilde{a} \in \widetilde{S}_{\text {hom }}^{d, v}$ and $a_{\text {smooth }} \in S_{\text {hom }}^{d}$, choose

$$
p(\xi ; \mu)=\tilde{\chi}(\xi) \widetilde{a}(\xi ; \mu)+\chi(\xi, \mu) a_{\text {smooth }}(\xi ; \mu)
$$

with arbitrary zero-excision functions $\chi(\xi, \mu)$ and $\tilde{\chi}(\xi)$. Changing the cut-off functions induces remainders in $S^{d-\infty, \nu-\infty}$; hence we may assume that $\tilde{\chi}(\xi) \chi(\xi, \mu)=\tilde{\chi}(\xi)$ and $p=\tilde{\chi} a+(1-\tilde{\chi}) \chi a_{\text {smooth }}$. Then taking another representation $a=\tilde{a}^{\prime}+a_{\text {smooth }}^{\prime}$ with associated symbol $p^{\prime}$, we find

$$
p-p^{\prime}=(1-\chi)(\xi) \kappa(\xi, \mu)\left(a_{\text {smooth }}-a_{\text {smooth }}^{\prime}\right) .
$$

Noting that (after restriction to the unit-sphere)

$$
a_{\text {smooth }}-a_{\text {smooth }}^{\prime}=r^{n(v)} b, \quad n(v)=\text { smallest integer } \geq v,
$$

with $b \in \mathscr{C}^{\infty}\left(\mathbb{S}_{+}^{n}\right)$ one concludes that $p-p^{\prime}$ belongs to $S^{d-\infty, n(v)-\infty} \subseteq S^{d-\infty, \nu-\infty}$.

In combination with Lemma 4.3 we obtain the following:

Theorem 4.6 Let $V=\{(\xi, \mu)|\mu \geq c| \xi \mid\}$ with some constant $c \geq 0$. Then

$$
S^{d, v}=\widetilde{S}_{V}^{d, v}+S^{d}
$$

where $\widetilde{S}_{V}^{d, v} \subset \widetilde{S}^{d, v}$ is the subspace of those symbols whose homogeneous components have support in $V$.

\section{Expansion at infinity}

One of the motivations for this paper is to extend the concept of ellipticity in the spaces $S^{d, v}$ with positive regularity number $v$ to the case $v=0$. Ellipticity should still be characterized by the invertibility of one or more principal symbols (plus some uniformity assumptions for preserving the $\mathscr{C}_{b}^{\infty}$ structure in $\left.x\right)$ and should imply invertibility of $a(x, D ; \mu)$ for large values of the parameter $\mu$. Recall that $S^{d, 0}=\widetilde{S}^{d, 0}$; for systematic reasons we address this question in $\widetilde{S}^{d, \nu}$ for arbitrary $\nu$.

In a first step, in Sect. 5.1, we introduce a subclass $\widetilde{\mathbf{S}}_{1,0}^{d, v}$ of $\widetilde{S}_{1,0}^{d, v}$ in which elliptic elements are invertible for large values of $\mu$. The ellipticity involves an estimate of the full symbol and the invertibility of a so-called limit-symbol; the latter plays the role of a new principal symbol. In a second step, we pass to the subclass of poly-homogeneous symbols $\widetilde{\mathbf{S}}^{d, v}$ where the full symbol can be replaced by the homogeneous principal symbol. 


\subsection{Symbols with expansion at infinity}

Definition 5.1 We denote by $\widetilde{\mathbf{S}}_{1,0}^{d, v}, d, v \in \mathbb{R}$, the subspace of $\widetilde{S}_{1,0}^{d, v}$ consisting of all symbols $a(x, \xi ; \mu)$ for which exists a sequence of symbols $a_{[v+j]}^{\infty} \in S_{1,0}^{\nu+j}\left(\mathbb{R}^{n}\right), j \in \mathbb{N}_{0}$, such that

$$
r_{a, N}(x, \xi ; \mu):=a(x, \xi ; \mu)-\sum_{j=0}^{N-1} a_{[\nu+j]}^{\infty}(x, \xi)[\xi, \mu]^{d-v-j} \in \widetilde{S}_{1,0}^{d, v+N}
$$

for every $N \in \mathbb{N}_{0}$; here $[\xi, \mu] \in S^{1}$ denotes a smooth positive function that coincides with $|(\xi, \mu)|$ outside some compact set. The symbol $a_{[v]}^{\infty}$ shall be called the principal limit-symbol of $a$.

The definition does not depend on the choice of the function $[\xi, \mu]$, since the difference of two such functions belongs to $\mathscr{C}_{\text {comp }}^{\infty}\left(\overline{\mathbb{R}}_{+} \times \mathbb{R}^{n}\right)$; for further discussion see Sect. 7.2. The coefficients $a_{[v+j]}^{\infty}(x, \xi)$ are uniquely determined by $a . \widetilde{\mathbf{S}}_{1,0}^{d, v}$ is a Fréchet space when equipped with the projective topology with respect to the mappings

$$
a \mapsto r_{a, N}: \widetilde{\mathbf{S}}_{1,0}^{d, v} \longrightarrow \widetilde{S}_{1,0}^{d, v+N}, \quad a \mapsto a_{[v+j]}^{\infty}: \widetilde{\mathbf{S}}_{1,0}^{d, v} \longrightarrow S_{1,0}^{\nu+j}\left(\mathbb{R}^{n}\right) .
$$

Note that $\widetilde{\mathbf{S}}_{1,0}^{d-N, v-N} \subset \widetilde{\mathbf{S}}_{1,0}^{d, v}$ whenever $N \in \mathbb{N}$; we define

$$
\widetilde{\mathbf{S}}_{1,0}^{d-\infty, \nu-\infty}=\bigcap_{N \in \mathbb{N}} \widetilde{\mathbf{S}}_{1,0}^{d-N, v-N} .
$$

Obviously, the maps

$$
a \mapsto\langle\xi\rangle^{e} a: \widetilde{\mathbf{S}}_{1,0}^{d, v} \longrightarrow \widetilde{\mathbf{S}}_{1,0}^{d+e, v+e}, \quad a \mapsto[\xi, \mu]^{e} a: \widetilde{\mathbf{S}}_{1,0}^{d, v} \longrightarrow \widetilde{\mathbf{S}}_{1,0}^{d+e, v},
$$

are isomorphisms with $\left(\langle\xi\rangle^{e} a\right)_{[v+e+j]}^{\infty}=\langle\xi\rangle^{e} a_{[v+j]}^{\infty}$ and $\left([\xi, \mu]^{e} a\right)_{[v+j]}^{\infty}=a_{[v+j]}^{\infty}$.

Example 5.2 Let $a(x, \xi) \in S_{1,0}^{d}\left(\mathbb{R}^{n}\right)$ be independent of $\mu$. Then $a \in \widetilde{\mathbf{S}}_{1,0}^{d, d}$ with $a_{[d]}^{\infty}=a$ and $a_{[d+j]}^{\infty}=0$ for every $j \geq 1$.

Proposition 5.3 Let $a \in S^{d}$. Then $a \in \widetilde{\mathbf{S}}_{1,0}^{d, 0}$ with principal limit-symbol

$$
a_{[0]}^{\infty}(x, \xi)=a_{0}(x, 0 ; 1),
$$

i.e., the homogeneous principal symbol of a evaluated in $(\xi, \mu)=(0,1)$. Moreover, $a_{[j]}^{\infty}(x, \xi)$ is a homogeneous polynomial in $\xi$ of order $j$.

Note that the proof of Proposition 5.3 is constructive, i.e., for given $a$ all symbols $a_{[j]}^{\infty}(x, \xi)$ can be calculated explicitly.

Proof of Proposition 5.3 For convenience assume independence on the $x$-variable. First note that $S^{d-N} \subseteq \widetilde{S}_{1,0}^{d-N, 0} \subseteq \widetilde{S}_{1,0}^{d, N}$, since

$$
\langle\xi, \mu\rangle^{d-N-|\alpha|-j} \leq\langle\xi\rangle^{-|\alpha|}\langle\xi, \mu\rangle^{d-N-j} \leq\langle\xi\rangle^{N-|\alpha|}\langle\xi, \mu\rangle^{d-N-j} .
$$

Thus we may assume that $a(\xi ; \mu)=\chi(\xi, \mu) a_{\ell}(\xi ; \mu)$ with $a_{\ell} \in S_{\text {hom }}^{d-\ell}, \ell \geq 0$, and a zeroexcision function $\chi(\xi, \mu)$, and show that $a$ belongs to $\widetilde{\mathbf{S}}_{1,0}^{d-\ell, 0} \subseteq \widetilde{\mathbf{S}}_{1,0}^{d, 0}$.

Let $\widehat{\kappa} \in \mathscr{C}^{\infty}\left(\mathbb{S}_{+}^{n}\right)$ be supported close to $(0,1)$ and $\kappa \equiv 1$ near $(0,1)$ and define $\kappa(\xi ; \mu):=$ $\widehat{\kappa}((\xi, \mu) /|\xi, \mu|)$. 
Step 1: $1-\kappa$ is supported in a conical set $V$ of the form $V=\{(\xi, \mu)|| \xi \mid \geq c \mu\}$ with $c>0$. Therefore, $(1-\kappa)(\xi ; \mu) a(\xi ; \mu) \in \widetilde{S}^{d-\ell, L}$ for every $L$, since $\langle\xi\rangle \sim\langle\xi, \mu\rangle$ on its support, hence $\langle\xi, \mu\rangle^{d-\ell-|\alpha|-j} \sim\langle\xi\rangle^{L-|\alpha|}\langle\xi, \mu\rangle^{d-\ell-L-j}$.

Step 2: Assume that $\left.a_{\ell}\right|_{\mathbb{S}_{+}^{n}}$ vanishes to order $N$ in $(\xi, \mu)=(0,1)$. Then

$$
u(\xi):=\left(\kappa a_{\ell}\right)\left(\xi ; \sqrt{1-|\xi|^{2}}\right)
$$

is a smooth function with compact support in $B:=\{\xi|| \xi \mid<1\}$ that vanishes to order $N$ in $\xi=0$. Write $u(\xi)=\sum_{|\alpha|=N} \xi^{\alpha} u_{\alpha}(\xi)$ with $u_{\alpha}$ also compactly supported in $B$. Then

$$
\left(\kappa a_{\ell}\right)(\xi ; \mu)=|\xi, \mu|^{d-\ell} u(\xi /|\xi, \mu|)=|\xi, \mu|^{d-\ell-N} \sum_{|\alpha|=N} \xi^{\alpha} u_{\alpha}(\xi /|\xi, \mu|)
$$

and therefore

$$
(\kappa a)(\xi ; \mu)=\sum_{|\alpha|=N} \xi^{\alpha} p_{\alpha}(\xi ; \mu), \quad p_{\alpha} \in S^{d-\ell-N} .
$$

Hence $(\kappa a)(\xi ; \mu) \in \widetilde{S}_{1,0}^{d-\ell, N}$ and thus, by Step $1, a(\xi ; \mu) \in \widetilde{S}_{1,0}^{d-\ell, N}$.

Step 3: Let $p(\xi ; \mu)=\sum_{|\alpha| \leq N-1} u_{\alpha} \xi^{\alpha}[\xi, \mu]^{d-\ell-|\alpha|}$ where $u_{\alpha}$ is the $\alpha$-th Taylor coefficient of $a_{\ell}\left(\xi ; \sqrt{1-|\xi|^{2}}\right)$ in $\xi=0$. Then $p \in S^{d-\ell}$ is homogeneous of degree $d-\ell$ for $|\xi, \mu| \geq 1$; let $p_{\ell} \in S_{\text {hom }}^{d-\ell}$ be the homogeneous principal symbol. Write

$$
a-p=\chi\left(a_{\ell}-p_{\ell}\right)-\left(p-\chi p_{\ell}\right)=\chi\left(a_{\ell}-p_{\ell}\right) \bmod S^{-\infty} .
$$

Since $\left.\left(a_{\ell}-p_{\ell}\right)\right|_{\mathbb{S}_{+}^{n}}$ vanishes to order $N$ in $(0,1)$, we conclude by Step 2 that $a-p \in \widetilde{S}_{1,0}^{d-\ell, N}$. Hence

$$
a(\xi ; \mu) \equiv \sum_{j=0}^{N-1} a_{[j]}^{\infty}(\xi)[\xi, \mu]^{d-\ell-j}, \quad a_{[j]}^{\infty}(\xi)=\sum_{|\alpha|=j} u_{\alpha} \xi^{\alpha},
$$

modulo $\widetilde{S}_{1,0}^{d-\ell, N}$.

\section{Lemma 5.4 The following holds true:}

i) If $a_{k} \in \widetilde{\mathbf{S}}_{1,0}^{d_{k}, v_{k}}$ for $k=0,1$, then $a_{1} a_{0} \in \widetilde{\mathbf{S}}_{1,0}^{d_{0}+d_{1}, v_{0}+v_{1}}$ with

$$
\left(a_{1} a_{0}\right)_{\left[v_{0}+v_{1}+j\right]}^{\infty}=\sum_{k+\ell=j} a_{1,\left[\nu_{1}+\ell\right]}^{\infty} a_{0,\left[v_{0}+k\right]}^{\infty} .
$$

ii) $D_{\xi}^{\alpha} D_{x}^{\beta}: \widetilde{\mathbf{S}}_{1,0}^{d, v} \rightarrow \widetilde{\mathbf{S}}_{1,0}^{d-|\alpha|, v-|\alpha|}$ with $\left(D_{\xi}^{\alpha} D_{x}^{\beta} a\right)_{[v-|\alpha|]}^{\infty}=D_{\xi}^{\alpha} D_{x}^{\beta} a_{[v]}^{\infty}$,

iii) $\partial_{\mu}^{j}: \widetilde{\mathbf{S}}_{1,0}^{d, v} \rightarrow \widetilde{\mathbf{S}}_{1,0}^{d-j, v}$ with $\left(\partial_{\mu}^{j} a\right)_{[v]}^{\infty}=(d-v)(d-v-1) \ldots(d-v-j+1) a_{[v]}^{\infty}$.

Proof i) is straight-forward, as is ii) using induction on $|\alpha|$.

By induction, it is enough to show iii) for $j=1$. Observe that

$$
\partial_{\mu}[\xi, \mu]^{d-v-j} \equiv(d-v-j)[\xi, \mu]^{d-v-j-2} \mu \bmod \mathscr{C}_{\text {comp }}^{\infty}\left(\overline{\mathbb{R}}_{+} \times \mathbb{R}^{n}\right) .
$$

Now use the expansion of $\mu \in \widetilde{\mathbf{S}}_{1,0}^{1,0}$, cf. Proposition 5.3, to find a resulting expansion of $\partial_{\mu} a$. 
Theorem 5.5 (Asymptotic summation) Let $a_{j} \in \widetilde{\mathbf{S}}_{1,0}^{d-j, \nu-j}, j \in \mathbb{N}_{0}$. Then there exists an $a \in \widetilde{\mathbf{S}}_{1,0}^{d, v}$ such that $a-\sum_{j=0}^{N-1} a_{j} \in \widetilde{\mathbf{S}}_{1,0}^{d-N, v-N}$ for every N. Moreover,

$$
a_{[v+j]}^{\infty} \sim \sum_{k=0}^{+\infty} a_{k,[(\nu-k)+j]}^{\infty}, \quad j \in \mathbb{N}_{0},
$$

asymptotically in $S_{1,0}^{\nu+j}\left(\mathbb{R}^{n}\right)$. The symbol a is unique modulo $\widetilde{\mathbf{S}}_{1,0}^{d-\infty, \nu-\infty}$.

Proof Let $\chi(\xi)$ be a zero-excision function and denote by $\chi_{c}, c>0$, the operator of multiplication by $\chi(\xi / c)$. Then $\chi_{c} \in \mathscr{L}\left(\widetilde{\mathbf{S}}_{1,0}^{d, v}\right)$ for every $d$, v with $\left(\chi_{c} a\right)_{[v+j]}^{\infty}=\chi_{c} a_{[v+j]}^{\infty}$ and $\left(1-\chi_{c}\right) a \in \widetilde{\mathbf{S}}_{1,0}^{d-\infty, \nu-\infty}$.

Moreover, the following statements are checked by straight-forward calculations:

(1) If $a \in \widetilde{S}_{1,0}^{d-1, v-1}$ then $\chi_{c} a \stackrel{c \rightarrow+\infty}{\longrightarrow} 0$ in $\widetilde{S}_{1,0}^{d, v}$.

(2) If $a \in \widetilde{\mathbf{S}}_{1,0}^{d-1, v-1}$ then $\chi_{c} a_{[v-1+j]}^{\infty} \stackrel{c \rightarrow+\infty}{\longrightarrow} 0$ in $S_{1,0}^{\nu+j}\left(\mathbb{R}^{n}\right)$.

(3) If $r \in \widetilde{S}_{1,0}^{d-1, \nu-1+N}$ then $\chi_{c} r \stackrel{c \rightarrow+\infty}{\longrightarrow} 0$ in $\widetilde{S}_{1,0}^{d, \nu+N}$.

In other words, given $a \in \widetilde{\mathbf{S}}_{1,0}^{d-1, v-1}$ then $\chi_{c} a \stackrel{c \rightarrow+\infty}{\longrightarrow} 0$ in $\widetilde{\mathbf{S}}_{1,0}^{d, \nu}$.

Now the existence of $a$ follows from Proposition 1.1.17 of [11] (with $E^{j}:=\widetilde{\mathbf{S}}_{1,0}^{d-j, \nu-j}$ and $\left.\chi^{j}(c)=\chi_{c}: E^{j} \rightarrow E^{j}\right)$. The remaining statements are clear.

For the detailed proofs of the following two theorems, concerning composition and (formal) adjoint, see the appendix.

Theorem 5.6 Let $a_{j} \in \widetilde{\mathbf{S}}_{1,0}^{d_{j}, v_{j}}, j=0$, 1. Then $a_{1} \# a_{0} \in \widetilde{\mathbf{S}}_{1,0}^{d_{0}+d_{1}, v_{0}+v_{1}}$ and the limit-symbol behaves multiplicatively: $\left(a_{1} \# a_{0}\right)_{\left[v_{0}+v_{1}\right]}^{\infty}=a_{1,\left[v_{1}\right]}^{\infty} \# a_{0,\left[v_{0}\right]}^{\infty}$. Moreover,

$$
a_{1} \# a_{0} \equiv \sum_{|\alpha|=0}^{N-1} \frac{1}{\alpha !}\left(\partial_{\xi}^{\alpha} a_{1}\right)\left(D_{x}^{\alpha} a_{0}\right) \quad \bmod \widetilde{\mathbf{S}}_{1,0}^{d_{0}+d_{1}-N, \nu_{0}+v_{1}-N} .
$$

Theorem 5.7 If $a \in \widetilde{\mathbf{S}}_{1,0}^{d, v}$ then $a^{(*)} \in \widetilde{\mathbf{S}}_{1,0}^{d, v}$ with $\left(a^{(*)}\right)_{[v]}^{\infty}=\left(a_{[v]}^{\infty}\right)^{(*)}$ and

$$
a^{(*)}(x, \xi ; \mu)=\sum_{|\alpha|=0}^{N-1} \frac{1}{\alpha !} \partial_{\xi}^{\alpha} D_{x}^{\alpha} \overline{a(x, \xi ; \mu)} \quad \bmod \widetilde{\mathbf{S}}_{1,0}^{d-N, \nu-N} .
$$

\subsection{Ellipticity and parametrix construction}

For the following considerations it is convenient to introduce the spaces $S_{1,0}^{d}\left(\overline{\mathbb{R}}_{+} ; E\right)$ consisting of all smooth functions $a(\mu)$ with values in a Frèchet space $E$, such that

$$
\left\|D_{\mu}^{j} a(\mu)\right\| \mid \lesssim\langle\mu\rangle^{d-j}
$$

for every $j$ and every continuous semi-norm $\||\cdot|\| \mid$ of $E$.

Lemma 5.8 Let $a(x, \xi ; \mu) \in \widetilde{\mathbf{S}}_{1,0}^{0-\infty, 0-\infty}$ and assume that $1-a_{[0]}^{\infty}(x, D)$ is invertible in $\mathscr{L}\left(H^{s}\left(\mathbb{R}^{n}\right)\right)$ for some $s \in \mathbb{R}$. Then $1-a(x, D ; \mu)$ is invertible for large $\mu$ and $(1-a(x, D ; \mu))^{-1}=1-b(x, D ; \mu)$ for some $b(x, \xi ; \mu) \in \widetilde{\mathbf{S}}_{1,0}^{0-\infty, 0-\infty}$. 
Proof First observe that $\widetilde{\mathbf{S}}_{1,0}^{0-\infty, 0-\infty}$ consists of those symbols $a$ for which exists a sequence of symbols $a_{[j]}^{\infty} \in S^{-\infty}\left(\mathbb{R}^{n}\right)$ such that, for every $N \in \mathbb{N}_{0}$,

$$
a(x, \xi ; \mu)=\sum_{j=0}^{N-1} a_{[j]}^{\infty}(x, \xi)[\xi, \mu]^{-j} \bmod \widetilde{S}_{1,0}^{0-\infty, N-\infty}=S_{1,0}^{-N}\left(\overline{\mathbb{R}}_{+}, S^{-\infty}\left(\mathbb{R}^{n}\right)\right) .
$$

Also recall that $(1-T)^{-1}=\sum_{j=0}^{N-1} T^{j}+T^{N}(1-T)^{-1}$ whenever $T$ belongs to a unital algebra and $1-T$ is invertible.

Step 1: Let us assume that $a_{[0]}^{\infty}=0$. In particular, $a \in S_{1,0}^{-1}\left(\overline{\mathbb{R}}_{+}, S^{-\infty}\left(\mathbb{R}^{n}\right)\right)$.

Due to the spectral-invariance of the algebra $\left\{p(x, D) \mid p \in S_{1,0}^{0}\left(\mathbb{R}^{n}\right)\right\}$ in $\mathscr{L}\left(H^{s}\left(\mathbb{R}^{n}\right)\right)$, we find that $1-a$ is invertible with respect to the Leibniz product for large $\mu$ and that $\chi(\mu)(1-a(\mu))^{-\#}$ belongs to $S_{1,0}^{0}\left(\overline{\mathbb{R}}_{+}, S^{0}\left(\mathbb{R}^{n}\right)\right)$ for a suitable zero-excision function $\chi$. But then

$$
b:=-a-a \# \chi(1-a)^{-\#} \# a \in S_{1,0}^{-1}\left(\overline{\mathbb{R}}_{+}, S^{-\infty}\left(\mathbb{R}^{n}\right)\right)
$$

and $1-b=(1-a)^{-\#}$ for large $\mu$. Hence, for large $\mu$,

$$
(1-a)^{-\#}=1+\sum_{j=1}^{N-1} a^{\# j}+a^{\# N} \#(1-b) \equiv 1+\sum_{j=1}^{N-1} a^{\# j} \quad \bmod S_{1,0}^{-N}\left(\overline{\mathbb{R}}_{+}, S^{-\infty}\left(\mathbb{R}^{n}\right)\right) .
$$

Using the expansions of $a^{\# j} \in \widetilde{\mathbf{S}}_{1,0}^{0-\infty, 0-\infty}$ and noting that $\left(a^{\# j}\right)_{[0]}^{\infty}=0$ for every $j$ due to the multiplicativity of the principal limit-symbol, we find a sequence of symbols $b_{[j]}^{\infty} \in S^{-\infty}\left(\mathbb{R}^{n}\right)$ such that, for every $N \in \mathbb{N}_{0}$,

$$
(1-a)^{-\#}=1+\sum_{j=1}^{N-1} b_{[j]}^{\infty}[\xi, \mu]^{-j}+r_{N}, \quad r_{N} \in S_{1,0}^{-N}\left(\overline{\mathbb{R}}_{+}, S^{-\infty}\left(\mathbb{R}^{n}\right)\right),
$$

for large $\mu$. Thus, for a suitable zero-excision function $\kappa(\mu)$,

$$
1-b=\kappa(1-b)+(1-\kappa)-(1-\kappa) b \equiv \kappa(1-a)^{-\#}+(1-\kappa) \bmod S^{-\infty},
$$

hence

$$
1-b \equiv 1+\kappa \sum_{j=1}^{N-1} b_{[j]}^{\infty}[\xi, \mu]^{-j}+\kappa r_{N} \equiv 1+\sum_{j=1}^{N-1} b_{[j]}^{\infty}[\xi, \mu]^{-j},
$$

modulo $S_{1,0}^{-N}\left(\overline{\mathbb{R}}_{+}, S^{-\infty}\left(\mathbb{R}^{n}\right)\right)$, since $(1-\kappa) b_{[j]}^{\infty} \in S^{-\infty}$.

Step 2: In the general case, again by spectral invariance, we find a $b_{[0]}^{\infty} \in S^{-\infty}\left(\mathbb{R}^{n}\right)$ such that $1-b_{[0]}^{\infty}(x, D)$ is the inverse of $1-a_{[0]}^{\infty}(x, D)$. Then $(1-a) \#\left(1-b_{[0]}^{\infty}\right)=1-a^{\prime}$, where $a^{\prime} \in \widetilde{\mathbf{S}}_{1,0}^{0-\infty, 0-\infty}$ has vanishing principal limit-symbol. Apply Step 1 to $1-a^{\prime}$ to find a corresponding parametrix $1-b^{\prime}$. Then the claim follows by choosing $b=1-\left(1-b_{[0]}^{\infty}\right) \#(1-$ $\left.b^{\prime}\right)=b^{\prime}+b_{[0]}^{\infty}-b_{[0]}^{\infty} \# b^{\prime}$.

Definition 5.9 We call $a \in \widetilde{\mathbf{S}}_{1,0}^{d, v}$ elliptic if there exist an $R \geq 0$ such that

(1) $a(x, \xi ; \mu)$ is invertible whenever $|\xi| \geq R$ and

$$
\left|a(x, \xi ; \mu)^{-1}\right| \lesssim\langle\xi\rangle^{-v}\langle\xi, \mu\rangle^{\nu-d},
$$

(2) $a_{[v]}^{\infty}(x, D)$ is invertible in $\mathscr{L}\left(H^{s}\left(\mathbb{R}^{n}\right), H^{s-v}\left(\mathbb{R}^{n}\right)\right)$ for some $s \in \mathbb{R}$. 
Note that condition (2) is equivalent to the existence of an inverse of $a_{[v]}^{\infty}(x, \xi)$ with respect to the Leibniz product, with inverse belonging to $S_{1,0}^{-v}\left(\mathbb{R}^{n}\right)$.

Theorem 5.10 Let $a \in \widetilde{\mathbf{S}}_{1,0}^{d, v}$ be elliptic. Then there exists $a b \in \widetilde{\mathbf{S}}_{1,0}^{-d,-v}$ such that both $a \# b-1$ and $b \# a-1$ belong to $\mathscr{C}_{\mathrm{comp}}^{\infty}\left(\overline{\mathbb{R}}_{+}, S^{-\infty}\left(\mathbb{R}^{n}\right)\right)$. In particular, $b(x, D ; \mu)=a(x, D ; \mu)^{-1}$ provided $\mu$ is sufficiently large.

Proof By order reduction we may assume without loss of generality that $d=v=0$.

Step 1: Since $a_{[0]}^{\infty}(x, D)$ is invertible, $a_{[0]}^{\infty}$ is also elliptic. Thus we can choose a zeroexcision function $\chi(\xi)$ such that

$$
\chi(2 \xi) a(x, \xi ; \mu)^{-1} \in \widetilde{S}_{1,0}^{0,0}, \quad \widetilde{c}_{[0]}^{\infty}(x, \xi):=\chi(2 \xi) a_{[0]}^{\infty}(x, \xi)^{-1} \in S_{1,0}^{0}\left(\mathbb{R}^{n}\right),
$$

and $\chi(\xi) \chi(2 \xi)=\chi(\xi)$. Now define recursively,

$$
\widetilde{c}_{[j]}^{\infty}(x, \xi)=-\widetilde{c}_{[0]}^{\infty}(x, \xi) \sum_{\substack{k+\ell=j, \ell<j}} a_{[k]}^{\infty}(x, \xi) \widetilde{c}_{[\ell]}^{\infty}(x, \xi), \quad j \in \mathbb{N},
$$

and set $c_{[j]}^{\infty}(x, \xi)=\chi(\xi) \tilde{c}_{[j]}^{\infty}(x, \xi)$. Then

$$
\left(\sum_{j=0}^{N-1} a_{[j]}^{\infty}(x, \xi)[\xi, \mu]^{-j}\right)\left(\sum_{j=0}^{N-1} c_{[j]}^{\infty}(x, \xi)[\xi, \mu]^{-j}\right)=\chi(\xi)-r_{N}(x, \xi ; \mu)
$$

with $r_{N} \in \widetilde{S}_{1,0}^{0, N}$. Thus, if $c(x, \xi ; \mu):=\chi(\xi) a(x, \xi ; \mu)^{-1}$, then

$$
\begin{aligned}
c(x, \xi ; \mu) & =\chi(\xi) \chi(2 \xi) a(x, \xi ; \mu)^{-1} \\
& \equiv\left(\sum_{j=0}^{N-1} a_{[j]}^{\infty}(x, \xi)[\xi, \mu]^{-j}\right)\left(\sum_{j=0}^{N-1} c_{[j]}^{\infty}(x, \xi)[\xi, \mu]^{-j}\right) \chi(2 \xi) a(x, \xi ; \mu)^{-1}
\end{aligned}
$$

modulo $\widetilde{S}_{1,0}^{0, N}$. The first factor on the right-hand side equals $a-r_{a, N}$ with $r_{a, N} \in \widetilde{S}^{0, N}$. It follows that

$$
c(x, \xi ; \mu) \equiv \sum_{j=0}^{N-1} c_{[j]}^{\infty}(x, \xi)[\xi, \mu]^{-j} \bmod \widetilde{S}_{1,0}^{0, N} .
$$

This shows that $c(x, \xi ; \mu)=\chi(\xi) a(x, \xi ; \mu)^{-1} \in \widetilde{\mathbf{S}}_{1,0}^{0,0}$.

Step 2: Let $c$ as constructed in Step 1. Then, by Theorem 5.6, $a \# c \equiv a c=\chi(\xi)$ modulo $\widetilde{\mathbf{S}}_{1,0}^{-1,-1}$. Thus $a \# c-1 \in \widetilde{\mathbf{S}}_{1,0}^{-1,-1}$ and the usual Neumann series argument, which is possible in view of Theorem 5.5, allows to construct a symbol $c^{\prime} \in \widetilde{\mathbf{S}}_{1,0}^{0,0}$ such that $a \# c^{\prime}=1-r$ with $r \in \widetilde{\mathbf{S}}_{1,0}^{0-\infty, 0-\infty}$. Now define

$$
c^{\prime \prime}:=c^{\prime}+\left(a_{[0]}^{\infty}\right)^{-\#} \# r_{[0]}^{\infty}
$$

note that $r_{[0]}^{\infty} \in S^{-\infty}\left(\mathbb{R}^{n}\right)$, hence $c^{\prime \prime}-c^{\prime} \in \widetilde{\mathbf{S}}_{1,0}^{0-\infty, 0-\infty}$. It follows that $a \# c^{\prime \prime}-1 \in \widetilde{\mathbf{S}}_{1,0}^{0-\infty, 0-\infty}$ and

$$
\left(a \# c^{\prime \prime}-1\right)_{[0]}^{\infty}=a_{[0]}^{\infty} \#\left(\left(c^{\prime}\right)_{[0]}^{\infty}+\left(a_{[0]}^{\infty}\right)^{-1} \# r_{[0]}^{\infty}\right)-1=a_{[0]}^{\infty} \#\left(c^{\prime}\right)_{[0]}^{\infty}+r_{[0]}^{\infty}-1=0
$$


by construction. Thus $a \# c^{\prime \prime}=1-r^{\prime}$, where $r^{\prime} \in \widetilde{\mathbf{S}}_{1,0}^{0-\infty, 0-\infty}$ has vanishing limit-symbol. Using Proposition 5.8 we thus find a right-parametrix $b_{R} \in \widetilde{\mathbf{S}}_{1,0}^{0,0}$ such that $a \# b_{R}-1 \in$ $\mathscr{C}_{\text {comp }}^{\infty}\left(\overline{\mathbb{R}}_{+}, S^{-\infty}\left(\mathbb{R}^{n}\right)\right)$.

Analogously, we construct a left-parametrix $b_{L}$. Then the claim follows by choosing $b=b_{L}$ or $b=b_{R}$.

\subsection{Poly-homogeneous symbols with expansion at infinity}

As already mentioned $\widetilde{S}_{h o m}^{d, v} \cong r^{v} \mathscr{C}_{B}^{\infty}\left(\widehat{\mathbb{S}}_{+}^{n}\right)$ does not behave well under inversion because there is no sufficient control at the singularity. We pass to a subclass which also is compatible with the previously introduced expansion at infinity.

In the following definition, we consider the north-pole $(0,1)$ as a singularity of the semisphere and consider functions having a particular asymptotic structure near this singularity. Asymptotics of this form are well-known in the context of manifolds with conical singularities, cf. for instance [11, Section 2.3].

Definition 5.11 A function $\widehat{a}(\xi ; \mu) \in r^{\nu} \mathscr{C}_{B}^{\infty}\left(\widehat{\mathbb{S}}_{+}^{n}\right)$ is said to have a weighted Taylor expansion (centered in the point $(0,1)$ ), if there exist $\widehat{a}_{\langle v+j\rangle} \in \mathscr{C}^{\infty}\left(\mathbb{S}^{n-1}\right), j \in \mathbb{N}_{0}$, such that the representation $\widehat{a}(r, \phi)=\widehat{a}\left(r \phi ; \sqrt{1-r^{2}}\right)$ of $a$ in polar-coordinates satisfies

$$
\widehat{a}(r, \phi)-\omega(r) \sum_{j=0}^{N-1} r^{\nu+j} \widehat{a}_{\langle\nu+j\rangle}(\phi) \in r^{\nu+N} \mathscr{C}_{B}^{\infty}\left((0,1), \mathscr{C}^{\infty}\left(\mathbb{S}^{n-1}\right)\right)
$$

for every $N \in \mathbb{N}_{0}$, where $\omega \in \mathscr{C}_{0}^{\infty}([0,1))$ is a cut-off function, i.e., $\omega$ has compact support in $[0,1)$ and $\omega \equiv 1$ near the origin. The space of all such functions $\widehat{a}(\xi ; \mu)$ will be denoted by $r^{v} \mathscr{C}_{T}^{\infty}\left(\widehat{\mathbb{S}}_{+}^{n}\right)$.

Note that $\widehat{a}(\xi ; \mu) \in r^{\nu} \mathscr{C}_{T}^{\infty}\left(\widehat{\mathbb{S}}_{+}^{n}\right)$ is invertible with inverse in $r^{-v} \mathscr{C}_{T}^{\infty}\left(\widehat{\mathbb{S}}_{+}^{n}\right)$ if, and only if, $\widehat{a}(\xi ; \mu) \neq 0$ whenever $\xi \neq 0$ and $\widehat{a}_{\langle v\rangle}(\phi) \neq 0$ for all $\phi \in \mathbb{S}^{n-1}$.

Definition 5.12 $\widetilde{\mathbf{S}}_{\text {hom }}^{d, v}$ consists of all functions of the form

$$
a(x, \xi ; \mu)=|\xi, \mu|^{d} \widehat{a}\left(x, \frac{(\xi, \mu)}{|\xi, \mu|}\right), \quad \widehat{a} \in \mathscr{C}_{b}^{\infty}\left(\mathbb{R}_{x}^{n}, r^{v} \mathscr{C}_{T}^{\infty}\left(\widehat{\mathbb{S}}_{+}^{n}\right)\right) .
$$

Define the principal angular symbol $a_{\langle\nu\rangle}(x, \xi) \in S_{h o m}^{\nu}\left(\mathbb{R}^{n}\right)$ (cf. Section 2.2) of $a$ as

$$
a_{\langle\nu\rangle}(x, \xi)=|\xi|^{v} \widehat{a}_{\langle v\rangle}\left(x, \frac{\xi}{|\xi|}\right)=|\xi|^{v} \lim _{r \rightarrow 0+} r^{-v} a\left(x, r \frac{\xi}{|\xi|} ; \sqrt{1-r^{2}}\right) .
$$

Note that, by construction, $\widetilde{\mathbf{S}}_{h o m}^{d, v} \subseteq \widetilde{S}_{h o m}^{d, v}$. The following proposition shows that such homogeneous components intrinsically admit an expansion at infinity in the sense of Definition 5.1.

Proposition 5.13 Let $a(x, \xi ; \mu) \in \widetilde{\mathbf{S}}_{\text {hom }}^{d, v}$ be as in Definition 5.12 with $\widehat{a}$ as in Definition 5.11. Let $p(x, \xi ; \mu)=\chi(\xi) a(x, \xi ; \mu)$ with a zero-excision function $\chi(\xi)$. Then $p \in \widetilde{\mathbf{S}}_{1,0}^{d, v}$ with

$$
p_{[v+j]}^{\infty}(x, \xi ; \mu)=\chi(\xi)|\xi|^{v+j} a_{\langle v+j\rangle}\left(x, \frac{\xi}{|\xi|}\right), \quad j \in \mathbb{N}_{0} .
$$


Proof By Theorem 4.4,

$$
\begin{aligned}
p(x, \xi ; \mu) & \equiv \chi(\xi)|\xi, \mu|^{d} \omega\left(\frac{|\xi|}{|\xi, \mu|}\right) \sum_{j=0}^{N-1}\left(\frac{|\xi|}{|\xi, \mu|}\right)^{v+j} a_{\langle v+j\rangle}\left(x, \frac{\xi}{|\xi|}\right) \\
& \equiv \chi(\xi) \omega\left(\frac{|\xi|}{|\xi, \mu|}\right) \sum_{j=0}^{N-1}|\xi|^{v+j} a_{\langle v+j\rangle}\left(x, \frac{\xi}{|\xi|}\right)[\xi, \mu]^{d-v-j}
\end{aligned}
$$

modulo $\widetilde{S}^{d, \nu+N}$ for every $N$. Now observe that $w(\xi ; \mu):=(1-\omega)\left(\frac{|\xi|}{|\xi, \mu|}\right)$ is a smooth function on $\left(\mathbb{R}^{n} \times \overline{\mathbb{R}}_{+}\right) \backslash\{0\}$ which is homogeneous of degree 0 and is supported in a set of the form $\{(\xi, \mu)|0 \leq \mu \leq c| \xi \mid\}$. Thus, if $\kappa(\xi, \mu)$ is a zero-excision function, then $\kappa(\xi, \mu) w(\xi ; \mu) \in \widetilde{S}_{1,0}^{0, L}$ for every $L$, since on its support $\langle\xi, \mu\rangle \sim\langle\xi\rangle$. Choosing $\kappa$ such that $\kappa(\xi, \mu) \chi(\xi)=\chi(\xi)$, we conclude that

$$
p(\xi ; \mu) \equiv \sum_{j=0}^{N-1} \chi(\xi)|\xi|^{v+j} a_{\langle\nu+j\rangle}\left(x, \frac{\xi}{|\xi|}\right)[\xi, \mu]^{d-v-j}
$$

modulo $\widetilde{S}^{d, v+N}$ for every $N$. This concludes the proof.

Definition 5.14 The space $\widetilde{\mathbf{S}}^{d, v}$ consists of all symbols $a(x, \xi ; \mu) \in \widetilde{\mathbf{S}}_{1,0}^{d, v}$ for which exists a sequence of homogeneous components $a_{j}(x, \xi ; \mu) \in \widetilde{\mathbf{S}}_{h o m}^{d-j, \nu-j}$ such that

$$
a(x, \xi ; \mu)-\chi(\xi) \sum_{j=0}^{N-1} a_{j}(x, \xi ; \mu) \in \widetilde{\mathbf{S}}_{1,0}^{d-N, \nu-N}
$$

for every $N \in \mathbb{N}_{0}$. The principal angular symbol of $a(x, \xi ; \mu)$ is, by definition, the principal angular symbol $a_{0,\langle v\rangle}(x, \xi)$ of the homogeneous principal symbol of $a_{0}(x, \xi ; \mu)$ (cf. Definition 5.12).

Due to Proposition 5.13 and Theorem 5.5, given any sequence of homogeneous components $a_{j}(x, \xi ; \mu) \in \widetilde{\mathbf{S}}_{h o m}^{d-j, v-j}$, there exists an $a \in \widetilde{\mathbf{S}}^{d, v}$ satisfying (5.3). If $a$ is as in (5.3) then the principal limit-symbol $a_{[\nu]}^{\infty}(x, \xi)$ belongs to $S^{\nu}\left(\mathbb{R}^{n}\right)$ and has the asymptotic expansion

$$
a_{[v]}^{\infty}(x, \xi) \sim \chi(\xi) \sum_{j=0}^{+\infty}|\xi|^{\nu-j} a_{j,\langle v-j\rangle}\left(x, \frac{\xi}{|\xi|}\right) .
$$

In particular, we have the following:

Proposition 5.15 Let $a(x, \xi ; \mu) \in \widetilde{\mathbf{S}}^{d, v}$. Then the homogeneous principal symbol of the principal limit-symbol $a_{[v]}^{\infty}(x, \xi)$ coincides with the principal angular symbol of $a(x, \xi ; \mu)$.

Now let us turn to ellipticity and parametrix.

Definition 5.16 A symbol $a(x, \xi ; \mu) \in \widetilde{\mathbf{S}}^{d, v}$ is called elliptic if

(1) The homogeneous principal symbol $a_{0}(x, \xi ; \mu)$ is invertible whenever $\xi \neq 0$ and

$$
\left|a_{0}(x, \xi ; \mu)^{-1}\right| \lesssim|\xi|^{-v}|\xi, \mu|^{\nu-d} \text {. }
$$

(2) $a_{[v]}^{\infty}(x, D)$ is invertible in $\mathscr{L}\left(H^{s}\left(\mathbb{R}^{n}\right), H^{s-v}\left(\mathbb{R}^{n}\right)\right)$ for some $s \in \mathbb{R}$. 
Due to Proposition 5.15, condition (2) implies the invertibility of the principal angular symbol of $a$. Moreover, if the homogeneous principal symbol of $a(x, \xi ; \mu)$ does not depend on $x$ for large $|x|$, then condition (1) in Definition 5.16 can be substituted by

$\left(1^{\prime}\right)$ The homogeneous principal symbol $a_{0}(x, \xi ; \mu)$ is invertible whenever $\xi \neq 0$.

Theorem 5.17 Let $a \in \widetilde{\mathbf{S}}^{d, v}$ be elliptic. Then there exists $a b \in \widetilde{\mathbf{S}}^{-d,-v}$ such that both $a \# b-1$ and $b \# a-1$ belong to $\mathscr{C}_{\text {comp }}^{\infty}\left(\overline{\mathbb{R}}_{+}, S^{-\infty}\left(\mathbb{R}^{n}\right)\right)$. In particular, $b(x, D ; \mu)=a(x, D ; \mu)^{-1}$ provided $\mu$ is sufficiently large.

Proof By ellipticity assumption (2), there exists a $b(x, \xi) \in S^{-v}\left(\mathbb{R}^{n}\right)$ which is the inverse of $a_{[v]}^{\infty}(x, \xi)$ with respect to the Leibniz product. By Proposition 5.15 it follows that the principal angular symbol of $a$ (i.e., that of $a_{0}$ ) is invertible and the inverse is just the homogeneous principal symbol of $b$. Together with (1) we conclude that the homogeneous principal symbol $a_{0}(x, \xi ; \mu)$ is invertible with inverse belonging to $\widetilde{\mathbf{S}}_{h o m}^{-d,-v}$. Thus there exists a $c(x, \xi ; \mu) \in$ $\widetilde{\mathbf{S}}^{-d,-v}$ which is a parametrix of $a(x, \xi ; \mu)$ modulo $\widetilde{\mathbf{S}}^{-1,-1}$. Then proceed as in Step 2 of the proof of Theorem 5.10.

\subsection{Refined calculus for symbols of finite regularity}

As proved in Theorem 4.6, Grubb's class $S^{d, v}$ coincides with the non-direct sum $\widetilde{S}^{d, v}+S^{d}$. In light of the above considerations it is now natural to introduce the following class:

Definition 5.18 With $d \in \mathbb{R}$ and $v \in \mathbb{Z}$ define

$$
\mathbf{S}^{d, v}=\widetilde{\mathbf{S}}^{d, v}+S^{d}, \quad \mathbf{S}_{h o m}^{d, v}=\widetilde{\mathbf{S}}_{h o m}^{d, v}+S_{h o m}^{d} .
$$

The limitation to integer values of $v$ is needed to ensure compatibility between the spaces $\widetilde{\mathbf{S}}_{\text {hom }}^{d, v}$ and $S_{\text {hom }}^{d}$ in the sense that the Taylor expansions (cf. Definition 5.11) associated with elements of either space only contain integer exponents; in particular, we have $\mathbf{S}^{d, v}=\widetilde{\mathbf{S}}^{d, v}$ whenever $v \leq 0$, and $\mathbf{S}^{d, v} \subset \widetilde{\mathbf{S}}^{d, 0}$ whenever $v>0$. The choice of integer $v$ is also important in view of Lemma 5.19.

By Proposition 5.3 and Theorem 5.6, the Leibniz product induces maps

$$
\mathbf{S}^{d_{1}, v_{1}} \times \mathbf{S}^{d_{0}, v_{0}} \longrightarrow \mathbf{S}^{d_{0}+d_{1}, v}, \quad v=\min \left(v_{0}, v_{1}, v_{0}+v_{1}\right) .
$$

By Theorem 5.7 the class is closed under taking the (formal) adjoint. Since in both spaces involved in Definition 5.18 asymptotic summation is possible (cf. Sect. 3.2 and Theorem 5.5), a sequence of symbols $a_{j} \in \mathbf{S}^{d-j, \nu-j}$ can be summed asymptotically in $\mathbf{S}^{d, v}$.

Lemma 5.19 Let $v$ be a positive integer ${ }^{1}$. Then the space $r^{v} \mathscr{C}_{T}^{\infty}\left(\widehat{\mathbb{S}}_{+}^{n}\right)+\mathscr{C}^{\infty}\left(\mathbb{S}_{+}^{n}\right)$ is closed under inversion, i.e., if $a(\xi ; \mu) \in r^{\nu} \mathscr{C}_{T}^{\infty}\left(\widehat{\mathbb{S}}_{+}^{n}\right)+\mathscr{C}^{\infty}\left(\mathbb{S}_{+}^{n}\right)$ is point-wise invertible everywhere on $\mathbb{S}_{+}^{n}$ then ${ }^{2} a(\xi ; \mu)^{-1} \in r^{\nu} \mathscr{C}_{T}^{\infty}\left(\widehat{\mathbb{S}}_{+}^{n}\right)+\mathscr{C}^{\infty}\left(\mathbb{S}_{+}^{n}\right)$.

Proof Write $a=\widehat{a}+a_{0}$ with $\widehat{a} \in r^{\nu} \mathscr{C}_{T}^{\infty}\left(\widehat{\mathbb{S}}_{+}^{n}\right)$ and $a_{0} \in \mathscr{C}^{\infty}\left(\mathbb{S}_{+}^{n}\right)$. Clearly $a$ is smooth on $\widehat{\mathbb{S}}_{+}^{n}$. We proceed in two steps:

Step 1: Let us assume that $a_{0} \equiv 1$ in some neighborhood of the point $(0,1)$. Choose $\psi_{1}, \psi \in \mathscr{C}^{\infty}\left(\mathbb{S}_{+}^{n}\right)$ having their support contained in this neighborhood and such that $\psi, \psi_{1} \equiv$

\footnotetext{
1 Actually, for the validity of this lemma it is sufficient to assume that $2 v$ is a positive integer.

2 Recall that $a(\xi ; \mu)$ extends by continuity to the whole semi-sphere, since $v>0$.
} 
1 near $(0,1)$ as well as $\psi_{1} \equiv 1$ on the support of $\psi$. Let $\widehat{b}=-\psi_{1} \widehat{a}$. Then $\widehat{b} \in r^{\nu} \mathscr{C}_{T}^{\infty}\left(\widehat{\mathbb{S}}_{+}^{n}\right)$ and, choosing $\psi_{1}$ with sufficiently small support, we have that $|\widehat{b}| \leq 1 / 2$ on $\mathbb{S}_{+}^{n}$, since $\lim _{(\xi, \mu) \rightarrow(0,1)} \widehat{a}(\xi ; \mu)=0$. Then

$$
\psi a^{-1}=\psi(1+\widehat{a})^{-1}=\psi\left(1+\psi_{1} \widehat{a}\right)^{-1}=\psi(1-\widehat{b})^{-1} .
$$

By chain rule it is straight-forward to see that $(1-\widehat{b})^{-1} \in \mathscr{C}_{B}^{\infty}\left(\widehat{\mathbb{S}}_{+}^{n}\right)$. Moreover,

$$
(1-\widehat{b})^{-1}=1+\sum_{j=1}^{L-1} \widehat{b}^{j}+\widehat{c}_{L}, \quad \widehat{c}_{L}:=\widehat{b}^{L}(1-\widehat{b})^{-1}, \quad L \in \mathbb{N} .
$$

Since $v$ is integer, $\widehat{b}^{j} \in r^{\nu} \mathscr{C}_{T}^{\infty}\left(\widehat{\mathbb{S}}_{+}^{n}\right)$ for every $j$. Inserting the Taylor expansion for each $\widehat{b}^{j}$ and noting that $\widehat{c}_{L} \in r^{v+N} \mathscr{C}_{B}^{\infty}\left(\widehat{\mathbb{S}}_{+}^{n}\right)$ provided $L=L(N)$ is taken large enough, we conclude that $(1-\widehat{b})^{-1}=1+\widehat{c}$ with $\widehat{c} \in r^{\nu} \mathscr{C}_{T}^{\infty}\left(\widehat{\mathbb{S}}_{+}^{n}\right)$. Therefore,

$$
a^{-1}=\psi a^{-1}+(1-\psi) a^{-1}=\psi \widehat{c}+\left(\psi+(1-\psi) a^{-1}\right) \in r^{\nu} \mathscr{C}_{T}^{\infty}\left(\widehat{\mathbb{S}}_{+}^{n}\right)+\mathscr{C}^{\infty}\left(\mathbb{S}_{+}^{n}\right) .
$$

Step 2: Consider the general case. Since $a_{0}(0 ; 1)=a(0 ; 1)$ is invertible, there exists a $b_{0} \in \mathscr{C}^{\infty}\left(\mathbb{S}_{+}^{n}\right)$ everywhere invertible and such that $b_{0}=a_{0}$ in a neighborhood of $(0,1)$. Then $a b_{0}^{-1} \in r^{\nu} \mathscr{C}_{T}^{\infty}\left(\widehat{\mathbb{S}}_{+}^{n}\right)+\mathscr{C}^{\infty}\left(\mathbb{S}_{+}^{n}\right)$ is everywhere invertible and $a b_{0}^{-1} \equiv 1$ near the point $(0,1)$. According to the first step, $b_{0} a^{-1}=\left(a b_{0}^{-1}\right)^{-1}$ belongs to $r^{\nu} \mathscr{C}_{T}^{\infty}\left(\widehat{\mathbb{S}}_{+}^{n}\right)+\mathscr{C}^{\infty}\left(\mathbb{S}_{+}^{n}\right)$. By multiplication with $b_{0}^{-1} \in \mathscr{C}^{\infty}\left(\mathbb{S}_{+}^{n}\right)$ we conclude that the same is true for $a^{-1}$.

In case of $x$-dependence we need to pose, as usual, an additional uniform bound on the inverse. Since symbols of $\mathbf{S}_{h o m}^{d, v}$ are just the homogeneous extensions of degree $d$ of functions from $r^{v} \mathscr{C}_{T}^{\infty}\left(\widehat{\mathbb{S}}_{+}^{n}\right)+\mathscr{C}^{\infty}\left(\mathbb{S}_{+}^{n}\right)$, we immediately have the following corollary.

Corollary 5.20 Let $v$ be a positive integer and $a(x, \xi ; \mu) \in \mathbf{S}_{\text {hom }}^{d, v}$. Assume that $a(x, \xi ; \mu)$ is invertible whenever $(\xi, \mu) \neq 0$ and that $\left|a(x, \xi ; \mu)^{-1}\right| \lesssim|\xi, \mu|^{-d}$. Then $a(x, \xi ; \mu)^{-1} \in$ $\mathbf{S}_{\text {hom }}^{-d, v}$.

After this observation it is clear that we can construct a parametrix in the class:

Theorem 5.21 Let $v$ be a positive integer and $a(x, \xi ; \mu) \in \mathbf{S}^{d, v}$ be elliptic (i.e., the homogeneous principal symbol satisfies the assumptions of Corollary 5.20). Then there exists a parametrix $b(x, \xi ; \mu) \in \mathbf{S}^{-d, v}$ such that both $a \# b-1$ and $b \# a-1$ belong to $\mathscr{C}_{\text {comp }}^{\infty}\left(\overline{\mathbb{R}}_{+}, S^{-\infty}\left(\mathbb{R}^{n}\right)\right)$.

If $a \in \mathbf{S}^{d, v}$ with positive integer $v$, then also $a \in \widetilde{\mathbf{S}}^{d, 0}$. Due to Propositions 5.13 and 5.3, its principal limit-symbol is

$$
a_{[0]}^{\infty}(x, \xi)=a_{0}(x, 0 ; 1),
$$

where $a_{0}$ is the homogeneous principal symbol of $a$ (defined on $\mathbb{S}_{+}^{n}$ by continuous extension). Recalling Definition 5.16, we find the following result which unifies the notions of ellipticity for symbols of regularity number $v=0$ and $v \in \mathbb{N}$, respectively.

Proposition 5.22 Let $v$ be a nonnegative integer and $a \in \mathbf{S}^{d, v}$. Then a is elliptic if, and only if, a is elliptic as an element of $\widetilde{\mathbf{S}}^{d, 0}$. 


\section{Resolvent-kernel expansions}

We shall discuss how our calculus allows to recover the well-known resolvent trace expansion for elliptic $\psi$ do due to Grubb-Seeley, cf. [6].

In the following we shall write $r(x, \xi ; \mu)=O\left(\mu^{m}, S_{1,0}^{M}\right)$ if $\mu^{-m} r(\mu) \in S_{1,0}^{M}\left(\mathbb{R}^{n}\right)$ uniformly in $\mu>0$.

\subsection{Preparation}

The following Lemma is a slight modification of [6, Lemma 1.3].

Lemma 6.1 Let $a(x, \xi ; \mu) \in S^{m}$ be homogeneous of degree $m$ for $|\xi, \mu| \geq 1$. Let $m_{+}=$ $\max (m, 0)$. Then there exist symbols $\zeta_{j}(x, \xi)=\sum_{|\alpha|=j} c_{j \alpha}(x) \xi^{\alpha}$ such that

$$
a(x, \xi ; \mu)=\sum_{j=0}^{N-1} \zeta_{j}(x, \xi) \mu^{m-j}+O\left(\mu^{m-N}, S_{1,0}^{m_{+}+N}\right)
$$

for every $N \in \mathbb{N}$. In particular, $\zeta_{0}(x, \xi)=a(x, 0 ; 1)$ and $\mu^{-m} a(x, \xi ; \mu) \rightarrow a(x, 0 ; 1)$ in $S_{1,0}^{m_{+}+1}\left(\mathbb{R}^{n}\right)$ as $\mu \rightarrow+\infty$.

Proof For convenience of notation assume independence of $x$. Obviously it suffices to consider $\mu \geq 1$. Then $a(\xi ; \mu)=\mu^{m} a(\xi / \mu ; 1)$. Let $u(t, \xi)=a(t \xi ; 1), 0 \leq t \leq 1$. The $j$-th $t$-derivative of $u$ is

$$
u^{(j)}(t, \xi)=\sum_{|\alpha|=j} c_{\alpha} \xi^{\alpha}\left(\partial_{\xi}^{\alpha} a\right)(t \xi ; 1)
$$

with certain universal constants $c_{\alpha}$. Thus the Taylor expansion of $u$ in $t$ centered in $t=0$ is of the form

$$
u(t, \xi)=\sum_{j=0}^{N-1} \zeta_{j}(\xi) t^{j}+t^{N} \int_{0}^{1}(1-\tau)^{N} u^{(N)}(t \tau, \xi) d \tau
$$

with polynomials $\zeta_{j}(\xi)$ as described. Then using the fact that

$$
\left|\partial_{\xi}^{\beta}\left[\left(\partial_{\xi}^{\alpha} a\right)(t \tau \xi ; 1)\right]\right| \lesssim(t \tau)^{|\beta|}\langle t \tau \xi\rangle^{m-|\alpha|-|\beta|} \lesssim(t \tau)^{|\beta|}\langle t \tau \xi\rangle^{-|\beta|}\langle\xi\rangle^{m_{+}} \lesssim\langle\xi\rangle^{m_{+}-|\beta|},
$$

for $0 \leq t, \tau \leq 1$, the above integral belongs to $S_{1,0}^{m_{+}+N}\left(\mathbb{R}^{n}\right)$ uniformly in $0 \leq t \leq 1$. Substituting $t=1 / \mu$ yields the claim.

A case of particular interest below is that

$$
[\xi, \mu]^{m}=\sum_{j=0}^{N-1} \zeta_{m, j}(\xi) \mu^{m-j}+O\left(\mu^{m-N}, S_{1,0}^{N}\right)
$$

whenever $m \leq 0$; any $\zeta_{m, j}(\xi)$ is a homogeneous polynomial of degree $j$.

Corollary 6.2 Let $a(x, \xi ; \mu) \in \widetilde{\mathbf{S}}_{1,0}^{d, v}$ with $d-v \leq 0$ have the expansion

$$
a(x, \xi ; \mu)=\sum_{j=0}^{N-1} a_{[v+j]}^{\infty}(x, \xi)[\xi, \mu]^{d-v-j} \bmod \widetilde{S}_{1,0}^{d, v+N} .
$$


Then

$$
a(x, \xi ; \mu)=\sum_{\ell=0}^{N-1} q_{\ell}(x, \xi) \mu^{d-v-\ell}+O\left(\mu^{d-v-N}, S_{1,0}^{\nu+N}\right),
$$

where, with notation of (6.1),

$$
q_{\ell}(x, \xi)=\sum_{j+k=\ell} a_{[v+j]}^{\infty}(x, \xi) \zeta_{d-v-j, k}(\xi) \in S_{1,0}^{\nu+\ell}\left(\mathbb{R}^{n}\right) .
$$

Proof First note that for $r(x, \xi ; \mu) \in \widetilde{S}^{d, \nu+N}$,

$$
\left|\partial_{\xi}^{\alpha} \partial_{x}^{\beta} r(x, \xi ; \mu)\right| \lesssim\langle\xi\rangle^{\nu+N-|\alpha|}\langle\xi, \mu\rangle^{d-\nu-N},
$$

hence $r(\mu)=O\left(\mu^{d-\nu-N}, S^{\nu+N}\right)$. Inserting the expansions

$$
[\xi, \mu]^{d-v-j}=\sum_{k=0}^{N-j-1} \zeta_{d-v-j, k}(\xi) \mu^{d-v-j-k}+O\left(\mu^{d-\nu-N}, S_{1,0}^{N-j}\right)
$$

the result follows immediately.

Theorem 6.3 Let $a(x, \xi ; \mu) \in \widetilde{\mathbf{S}}^{d, v}$ with $d<-n$ and $d-v \leq 0$. Let

$$
k(x, y ; \mu)=\int e^{i(x-y) \xi} a(x, \xi ; \mu) d \xi
$$

the distributional kernel of $a(x, D ; \mu)$. Then there exist functions $c_{\ell}(x), c_{\ell}^{\prime}(x), c_{\ell}^{\prime \prime}(x), j \in \mathbb{N}_{0}$, which are continuous and bounded such that, for $\mu \rightarrow+\infty$,

$$
k(x, x ; \mu) \sim \sum_{j=0}^{+\infty} c_{j}(x) \mu^{d-j+n}+\sum_{\ell=0}^{+\infty}\left(c_{\ell}^{\prime}(x) \log \mu+c_{\ell}^{\prime \prime}(x)\right) \mu^{d-v-\ell} .
$$

Proof We follow closely the proof of [6, Theorem 2.1]. Let $N$ be fixed. Choose, and fix, a $J \in \mathbb{N}$ so large that

$$
v-J+N<-n
$$

and write

$$
a(x, \xi ; \mu)=\chi(\xi) \sum_{j=0}^{J-1} a_{j}(x, \xi ; \mu)+r(x, \xi ; \mu), \quad r \in \widetilde{\mathbf{S}}^{d-J, \nu-J},
$$

where $a_{j}(x, \xi ; \mu) \in \widetilde{\mathbf{S}}_{h o m}^{d-j, \nu-j}$ and $\chi$ is a zero-excision function such that $1-\chi$ is supported in the unit-ball centered in the origin.

By Corollary 6.2 (with $d, v$ replaced by $d-J, v-J$ ) we have

$$
r(x, \xi ; \mu)=\sum_{\ell=0}^{N-1} q_{\ell}(x, \xi) \mu^{d-\nu-\ell}+O\left(\mu^{d-\nu-N}, S_{1,0}^{\nu-J+N}\right)
$$

with $q_{\ell}(x, \xi) \in S_{1,0}^{\nu-J+\ell}\left(\mathbb{R}^{n}\right)$. Recalling (6.2), the associated kernel $k_{r}(x, y ; \mu)$ satisfies

$$
k_{r}(x, x ; \mu)=\sum_{\ell=0}^{N-1} c_{r, \ell}^{\prime \prime}(x) \mu^{d-v-\ell}+O\left(\mu^{d-v-N}\right) .
$$


Now let $k_{j}(x, y ; \mu)$ denote the kernel associated with $\chi(\xi) a_{j}(x, \xi ; \mu)$. Decompose $k_{j}(x, x ; \mu)=k_{j}^{(1)}(x, x ; \mu)+k_{j}^{(2)}(x, x ; \mu)$ with

$$
k_{j}^{(1)}(x, x ; \mu)=\int_{|\xi| \geq \mu} \chi(\xi) a_{j}(x, \xi ; \mu) d \xi .
$$

Then, for every $\mu \geq 1$, using the homogeneity of $a_{j}$,

$$
k_{j}^{(1)}(x, x ; \mu)=\mu^{d-j+n} \int_{|\xi| \geq 1} a_{j}(x, \xi ; 1) d \xi=c_{j}(x) \mu^{d-j+n} ;
$$

note that the integrand is bounded by $\langle\xi\rangle^{d-j}$, hence integrable since $d<-n$.

Next choose $L$ with $L \geq N$ and $L>J-1-n-v$ (i.e., $v-j+L>-n$ for every $j=0, \ldots, J-1$ ). Apply Corollary 6.2 (with $d, v$ replaced by $d-j, v-j$ ) to write

$$
\begin{aligned}
\chi(\xi) a_{j}(x, \xi ; \mu) & =\sum_{\ell=0}^{L-1} q_{j, \ell}(x, \xi) \mu^{d-v-\ell}+s_{j, L}(x, \xi ; \mu), \\
s_{j, L}(x, \xi ; \mu) & =O\left(\mu^{d-v-L}, S_{1,0}^{\nu-j+L}\right) ;
\end{aligned}
$$

by Proposition 5.19 (more precisely, the last formula in its proof) the symbols $q_{j, \ell}(x, \xi) \in$ $S_{1,0}^{\nu-j+\ell}\left(\mathbb{R}^{n}\right)$ are homogeneous of degree $v-j+\ell$ for $|\xi| \geq 1$. Thus $s_{j, L}(x, \xi ; \mu)$ is homogeneous of degree $d-j$ in $(\xi, \mu)$ for $|\xi| \geq 1$. We now write

$$
\begin{aligned}
k_{j}^{(2)}(x, x ; \mu) & =k_{j}^{(2 a)}(x, x ; \mu)+k_{j}^{(2 b)}(x, x ; \mu) \\
& =\int_{|\xi| \leq 1} \chi(\xi) a_{j}(x, \xi ; \mu) d \xi+\int_{1 \leq|\xi| \leq \mu} a_{j}(x, \xi ; \mu) d \xi .
\end{aligned}
$$

By (6.3) we obtain immediately that

$$
k_{j}^{(2 a)}(x, x ; \mu)=\sum_{\ell=0}^{L-1} c_{j, \ell}^{\prime \prime}(x) \mu^{d-\nu-\ell}+O\left(\mu^{d-\nu-L}\right) .
$$

By homogeneity for $|\xi| \geq 1$ of the $q_{j, \ell}$ and by using polar-coordinates,

$$
\int_{1 \leq|\xi| \leq \mu} q_{j, \ell}(x, \xi) d \xi= \begin{cases}c_{j, \ell}^{\prime}(x)\left(\mu^{v-j+\ell+n}-1\right) & : v-j+\ell+n \neq 0, \\ c_{j, \ell}^{\prime}(x) \log \mu & : v-j+\ell+n=0\end{cases}
$$

By the second line in (6.3) and the homogeneity of $s_{j, L}$,

$$
s_{j, L}(x, \xi ; \mu)=|\xi|^{d-j} s_{j, L}\left(x, \frac{\xi}{|\xi|} ; \frac{\mu}{|\xi|}\right)=O\left(\mu^{d-\nu-L}|\xi|^{\nu-j+L}\right), \quad|\xi| \geq 1 .
$$

If $s_{j, L}^{h}$ denotes the extension by homogeneity of $s_{j, L}$ from $|\xi| \geq 1$ to all $\xi \neq 0$ (defined by the second term in (6.4)), then

$$
s_{j, L}^{h}(x, \xi ; \mu)=O\left(\mu^{d-v-L}|\xi|^{\nu-j+L}\right), \quad \xi \neq 0 .
$$

Then

$$
\begin{aligned}
\int_{1 \leq|\xi| \leq \mu} s_{j, L}(x, \xi ; \mu) d \xi & =\int_{0 \leq|\xi| \leq \mu} s_{j, L}^{h}(x, \xi ; \mu) d \xi-\int_{0 \leq|\xi| \leq 1} s_{j, L}^{h}(x, \xi ; \mu) d \xi \\
& =c_{j, L}(x) \mu^{d-j+n}+O\left(\mu^{d-\nu-L}\right) .
\end{aligned}
$$

This yields the expansion of $k_{j}^{(2 b)}(x, x ; \mu)$ and completes the proof. 


\subsection{Application to the resolvent of a $\psi$ do}

Assume we are given two $\psi$ do, $p(x, \xi) \in S^{m}\left(\mathbb{R}^{n}\right)$ of positive integer order $m \in \mathbb{N}$ and $q(x, \xi) \in S^{\omega}\left(\mathbb{R}^{n}\right)$ with $\omega \in \mathbb{R}$. Moreover, let

$$
\Lambda=\left\{\mu e^{i \theta}|\mu \geq 0,0 \leq| \theta \mid \leq \Theta\right\}, \quad 0<\Theta<\pi,
$$

be a sector in the complex plane. Then, for every $\theta$,

$$
a_{\theta}(x, \xi ; \mu):=\mu^{m}-e^{-i \theta} p(x, \xi) \in \mathbf{S}^{m, m} .
$$

Note that $e^{i \theta} a_{\theta}\left(x, \xi ; r^{1 / m}\right)=r e^{i \theta}-p(x, \xi)$. Now assume that $a_{\theta}$ is elliptic, uniformly with respect to $\theta$, i.e.,

$$
\left|\left(\mu^{m}-e^{-i \theta} p_{0}(x, \xi)\right)^{-1}\right| \lesssim 1, \quad|\xi, \mu|=1,
$$

uniformly in $x \in \mathbb{R}^{n}$ and $0 \leq|\theta| \leq \Theta$. Using Theorem 5.21, there exists a $b_{\theta}(x, \xi ; \mu) \in$ $\mathbf{S}^{-m, m}$, depending uniformly on $\theta$, such that $a_{\theta}(x, D ; \mu)$ is invertible for large $\mu$ with $a_{\theta}(x, D ; \mu)^{-1}=b_{\theta}(x, D ; \mu)$. We then find, for every positive integer $\ell$,

$$
c_{\theta}(x, D ; \mu):=q(x, D)\left(\mu^{m} e^{i \theta}-p(x, D)\right)^{-\ell}=e^{-i \theta \ell} q(x, D) b_{\theta}(x, D ; \mu)^{\ell} .
$$

Note that the $\ell$-fold Leibniz product of $b_{\theta}$ belongs to $\mathbf{S}^{-m \ell, m}=\widetilde{\mathbf{S}}^{-m \ell, m}+S^{-m \ell}$. Since $S^{-m \ell} \subset \widetilde{\mathbf{S}}^{-m \ell, 0}$, we find that $c_{\theta}=c_{\theta}^{(1)}+c_{\theta}^{(2)}$ with

$$
c_{\theta}^{(1)}(x, \xi ; \mu) \in \widetilde{\mathbf{S}}^{\omega-m \ell, \omega+m}, \quad c_{\theta}^{(2)}(x, \xi ; \mu) \in \widetilde{\mathbf{S}}^{\omega-m \ell, \omega},
$$

with uniform dependence on $\theta$. If $\ell$ is so large that $\omega-m \ell<-n$, we can apply Theorem 6.3 to both $c_{\theta}^{(1)}$ and $c_{\theta}^{(2)}$. This is the key to obtain the following:

Theorem 6.4 With the above notation and assumptions, let $k(x, y ; \lambda)$ be the distributional kernel of $q(x, D)(\lambda-p(x, D))^{-\ell}$. Then there exist $\mathscr{C}_{b}^{\infty}$-functions $c_{j}(x), c_{j}^{\prime}(x), c_{j}^{\prime \prime}(x), j \in$ $\mathbb{N}_{0}$, such that

$$
k(x, x ; \lambda) \sim \sum_{j=0}^{+\infty} c_{j}(x) \lambda^{\frac{n+\omega-j}{m}-\ell}+\sum_{j=0}^{+\infty}\left(c_{j}^{\prime}(x) \log \lambda+c_{j}^{\prime \prime}(x)\right) \lambda^{-\ell-\frac{j}{m}},
$$

uniformly for $\lambda \in \Lambda$ with $|\lambda| \longrightarrow+\infty$. Moreover, $c_{j}^{\prime}=c_{j}^{\prime \prime} \equiv 0$ whenever $j$ is not an integer multiple of $m$.

Proof of Theorem 6.4 Applying Theorem 6.3 to both $c_{\theta}^{(1)}$ and $c_{\theta}^{(2)}$ one obtains an expansion

$$
k\left(x, x ; \mu^{m} e^{i \theta}\right) \sim \sum_{j=0}^{+\infty} \widetilde{c}_{j}(x, \theta) \mu^{n+\omega-j-m \ell}+\sum_{j=0}^{+\infty}\left(\widetilde{c}_{j}^{\prime}(x, \theta) \log \mu+\widetilde{c}_{j}^{\prime \prime}(x, \theta)\right) \mu^{-\ell m-j},
$$

for $\mu \rightarrow+\infty$, uniformly in $\theta$. Writing $\log \mu=\log \left(\mu e^{i \theta}\right)-i \theta, \mu^{a}=\left(\mu e^{i \theta}\right)^{a}\left(e^{-i \theta}\right)^{a}$, and substituting $\mu=r^{1 / m}$ yields expansion (6.5), but with coefficient functions depending on $\theta$. However, due to the holomorphy of the left-hand side (for fixed $x$ ), the coefficients must be constant in $\theta$ as shown in [6, Lemma 2.3].

To see that the coefficients $c_{j}^{\prime}$ and $c_{j}^{\prime \prime}$ vanish whenever $j$ is not an integer multiple of $\ell$, one needs to repeat the considerations from [6, Section 2.2] concerning the construction of the parametrix of $\mu^{m}-p(x, \xi)$. 


\section{Operators on manifolds}

We shall show that the various symbol classes introduced so far lead to corresponding operator-classes on smooth compact manifolds. In particular, we shall show that the expansion at infinity and the concept of principal limit-symbol extend to the global setting.

\subsection{Invariance under change of coordinates}

Let $\kappa: \mathbb{R}^{n} \rightarrow \mathbb{R}^{n}$ be a smooth change of coordinates and assume that $\partial_{j} \kappa_{k} \in \mathscr{C}_{b}^{\infty}\left(\mathbb{R}^{n}\right)$ for all $1 \leq j, k \leq n$, and that $\left|\operatorname{det} \kappa^{\prime}\right|$ is uniformly bounded from above and below by positive constants; here, $\kappa^{\prime}$ denotes the first derivative (Jacobian matrix) of $\kappa$. For an operator $A: \mathscr{S}\left(\mathbb{R}^{n}\right) \rightarrow \mathscr{S}\left(\mathbb{R}^{n}\right)$ its push-forward $\kappa_{*} A$ is defined by

$$
\left(\kappa_{*} A\right) u=[A(u \circ \kappa)] \circ \kappa^{-1}, \quad u \in \mathscr{S}\left(\mathbb{R}^{n}\right) .
$$

Its pull-back is $\kappa^{*} A:=\left(\kappa^{-1}\right)_{*} A$. If $A(\mu)$ is depending on a parameter $\mu$, pull-back and pushforward are defined in the same way, resulting in families $\kappa^{*} A(\mu)$ and $\kappa_{*} A(\mu)$, respectively. It is then well-known that the classes $S_{1,0}^{d}$ and $S^{d}$ are invariant under the change of coordinates $x=\kappa(y)$.

Theorem 7.1 The classes $\widetilde{S}_{1,0}^{d, v}, \widetilde{S}^{d, v}, \widetilde{\mathbf{S}}_{1,0}^{d, v}$, and $\widetilde{\mathbf{S}}^{d, v}$ are invariant under the change of coordinates $x=\kappa(y)$. In the classes of poly-homogeneous symbols, the homogeneous principal symbols satisfy the (usual) relation

$$
\left(\kappa_{*} a\right)_{0}(x, \xi ; \mu)=a_{0}\left(\kappa^{-1}(x), \kappa^{\prime}\left(\kappa^{-1}(x)\right)^{t} \xi ; \mu\right),
$$

where $\kappa^{\prime}(y)^{t}$ denotes the adjoint of the first derivative $\kappa^{\prime}(y)$.

Proof In Theorem 2.1.21 of [3] the invariance is shown for the classes $S_{1,0}^{d, v}$ and $S^{d, v}$. This includes the classes $\widetilde{S}_{1,0}^{d, v}$ and $\widetilde{S}^{d, v}$ for $v \leq 0$. If $v>0$, we choose a symbol $p(x, \xi) \in$ $S^{-v}\left(\mathbb{R}^{n}\right)$ which has inverse $q(x, \xi) \in S^{v}\left(\mathbb{R}^{n}\right)$ with respect to the Leibniz product. Given $a(x, \xi ; \mu) \in \widetilde{S}_{1,0}^{d, v}$, we find

$$
\kappa_{*} a=\kappa_{*}(a \# p) \# \kappa_{*} q \in \widetilde{S}_{1,0}^{d, v},
$$

since $a \# p \in \widetilde{S}_{1,0}^{d-v, 0}$. Analogously we argue for $\widetilde{S}^{d, \nu}$.

Next let $a(x, \xi ; \mu) \in \widetilde{\mathbf{S}}_{1,0}^{d, v}$ be as in Definition 5.1. The invariance follows from the observation that the classes $S_{1,0}^{\nu+j}\left(\mathbb{R}^{n}\right)$ and $\widetilde{S}_{1,0}^{d, \nu+N}$ are invariant, while $\kappa_{*}[\xi, \mu]^{d-v-j} \in S^{d-v-j} \subset$ $\widetilde{\mathbf{S}}_{1,0}^{d-\nu-j, 0}$ has a complete expansion due to Proposition 5.3. This allows to find the complete expansion of $\kappa_{*} a(x, \xi ; \mu)$. Using the formula for the asymptotic expansion of $\kappa_{*} a$, one sees that poly-homogeneous symbols remain poly-homogeneous.

Let us have a closer look to the homogeneous principal symbol of $a \in \widetilde{\mathbf{S}}^{d, v}$. For convenience of notation let us set $p(x, \xi ; \mu)=\left(\kappa_{*} a\right)_{0}(x, \xi ; \mu)$ and $\mathcal{K}(x)=\kappa^{\prime}\left(\kappa^{-1}(x)\right)^{t}$. To see that $p$ belongs to $\widetilde{\mathbf{S}}_{h o m}^{d, v}$ we write

$$
p(x, \xi ; \mu)=|\xi, \mu|^{d} \widehat{p}\left(x, \frac{(\xi, \mu)}{|\xi, \mu|}\right),
$$

where, in polar-coordinates,

$$
\widehat{p}(x, r, \phi)=p\left(x, r \phi, \sqrt{1-r^{2}}\right)=a\left(\kappa^{-1}(x), r \mathcal{K}(x) \phi, \sqrt{1-r^{2}}\right) .
$$


Introducing

$$
\begin{aligned}
n(x, r, \phi)^{2} & =\left|r \mathcal{K}(x) \phi, \sqrt{1-r^{2}}\right|^{2}=1-r^{2}\left(1-|\mathcal{K}(x) \phi|^{2}\right), \\
s(x, r, \phi) & =r|\mathcal{K}(x) \phi| / n(x, r, \phi), \\
\theta(x, \phi) & =\mathcal{K}(x) \phi /|\mathcal{K}(x) \phi|,
\end{aligned}
$$

we find

$$
\widehat{p}(x, r, \phi)=n^{d} \widehat{a}\left(\kappa^{-1}(x), s \theta, \sqrt{1-s^{2}}\right) .
$$

Noting that $n$ is smooth in $r$ up to $r=0$ and using the weighted Taylor-expansion of $\widehat{a}$, one finds that $\widehat{p}$ admits a weighted Taylor-expansion with principal angular symbol

$$
\begin{aligned}
\widehat{p}_{\langle\nu\rangle}(x, \phi) & =\lim _{r \rightarrow 0+} n^{d}(r / s)^{-v} s^{-v} \widehat{a}\left(\kappa^{-1}(x), s \theta, \sqrt{1-s^{2}}\right) \\
& =|\mathcal{K}(x) \phi|^{v} a_{\langle\nu\rangle}\left(\kappa^{-1}(x), \theta\right) .
\end{aligned}
$$

This results in the following observation:

Proposition 7.2 Let $a \in \widetilde{\mathbf{S}}^{d, v}$. The principal angular symbols of a and $\kappa_{*}$ a satisfy the relation

$$
\left(\kappa_{*} a\right)_{\langle\nu\rangle}(x, \xi)=a_{\langle v\rangle}\left(\kappa^{-1}(x), \kappa^{\prime}\left(\kappa^{-1}(x)\right)^{t} \xi\right) .
$$

In other words, the principal angular symbol transforms as a function on the cotangentbundle of $\mathbb{R}^{n}$.

Remark 7.3 In the above discussion we have focused on changes of coordinates defined on $\mathbb{R}^{n}$, satisfying certain growth conditions at infinity. This is the natural setting for symbols which are globally defined on $\mathbb{R}^{n}$. Alternatively, we could consider arbitrary diffeomorphisms $\kappa: U \rightarrow V$ with arbitrary open subsets $U, V$ of $\mathbb{R}^{n}$ and the push-forward of $\psi$ do of the form $\phi a(x, D ; \mu) \psi$ with $\phi, \psi \in \mathscr{C}_{\mathrm{comp}}^{\infty}(U)$. We would obtain a corresponding invariance property; the details are left to the reader.

The invariance under changes of coordinates permits to define corresponding classes for manifolds.

Definition 7.4 Let $M$ be a smooth closed manifold. With $\widetilde{L}_{1,0}^{d, v}=\widetilde{L}_{1,0}^{d, v}\left(M ; \overline{\mathbb{R}}_{+}\right)$we denote the space of all operator-families $A(\mu): \mathscr{C}^{\infty}(M) \rightarrow \mathscr{C}^{\infty}(M)$ with the following property: Given an arbitrary chart $\kappa: \Omega \subset M \rightarrow U \subset \mathbb{R}^{n}$ and arbitrary functions $\phi, \psi \in \mathscr{C}_{\text {comp }}^{\infty}(\Omega)$, the operator-family $\kappa_{*}(\phi A(\mu) \psi)$ defined by

$$
u \mapsto \kappa_{*}(\phi A(\mu) \psi) u=[\phi A(\mu)(\psi(u \circ \kappa))] \circ \kappa^{-1}, \quad u \in \mathscr{S}\left(\mathbb{R}^{n}\right),^{3}
$$

is a $\psi$ do with symbol from $\widetilde{S}^{d, v}$. Analogously, define the spaces $\widetilde{L}^{d, v}=\widetilde{L}^{d, v}\left(M ; \overline{\mathbb{R}}_{+}\right)$, $\widetilde{\mathbf{L}}_{1,0}^{d, v}=\widetilde{\mathbf{L}}_{1,0}^{d, v}\left(M ; \overline{\mathbb{R}}_{+}\right)$, and $\widetilde{\mathbf{L}}^{d, v}=\widetilde{\mathbf{L}}^{d, v}\left(M ; \overline{\mathbb{R}}_{+}\right)$.

In $\widetilde{\mathbf{L}}^{d, v}$ both homogeneous principal symbol and principal angular symbol are well defined functions on $\left(T^{*} M \backslash 0\right) \times \overline{\mathbb{R}}_{+}$and $T^{*} M \backslash 0$, respectively. Let us mention that $\widetilde{L}_{1,0}^{d-\infty, \nu-\infty}=$ $\widetilde{L}^{d-\infty, \nu-\infty}=S_{1,0}^{d-v}\left(\overline{\mathbb{R}}_{+}, L^{-\infty}(M)\right)$.

Proceeding as usual, one can show that any of the four classes is closed under composition and, after fixing an arbitrary Riemannian metric on $M$ which allows the definition of a corresponding space $L^{2}(M)$ of square integrable functions, under taking the formal adjoint:

3 In this definition, smooth functions with compact support in some open set are considered as functions on the whole ambient space after extension by zero. 
Theorem 7.5 Composition of operator-families induces a map $\widetilde{L}_{1,0}^{d_{1}, v_{1}} \times \widetilde{L}_{1,0}^{d_{0}, \nu_{0}} \rightarrow$ $\widetilde{L}_{1,0}^{d_{0}+d_{1}, \nu_{0}+v_{1}}$; taking the formal adjoint induces a map $\widetilde{L}_{1,0}^{d, v} \rightarrow \widetilde{L}_{1,0}^{d, v}$. Analogous results hold for the three other classes introduced in Definition 7.4.

For an alternative description of the operator-classes, let us choose a system of charts $\kappa_{i}: \Omega_{i} \rightarrow U_{i}, i=1, \ldots, m$, such that the $\Omega_{i}$ cover $M$; moreover let $\phi_{i}, \psi_{i} \in \mathscr{C}^{\infty}\left(\Omega_{i}\right)$ such that the $\phi_{i}$ are a partition of unity and $\psi_{i} \equiv 1$ in a neighborhood of the support of $\phi_{i}$. Then $\widetilde{L}_{1,0}^{d, v}$ consists of all operators of the form

$$
A(\mu)=\sum_{i=1}^{m} \kappa_{i}^{*}\left(\left(\phi_{i} \circ \kappa_{i}^{-1}\right) a_{i}(x, D ; \mu)\left(\psi_{i} \circ \kappa_{i}^{-1}\right)\right) \bmod \widetilde{L}_{1,0}^{d-\infty, \nu-\infty}
$$

with $a_{i} \in \widetilde{S}_{1,0}^{d, v}$. The analogous statement holds for the other classes.

\subsection{Complete expansion and limit operator}

The extension of the concept of complete expansion and principal limit-symbol to manifolds requires some additional analysis. The key is to show that the symbol $[\xi, \mu]^{\alpha}$ involved in the definition of $\widetilde{\mathbf{S}}^{d, v}$ can be replaced by other ones.

It is convenient to use the notation $\lambda^{\alpha}(\xi, \mu)=[\xi, \mu]^{\alpha}, \alpha \in \mathbb{R}$. Then the expansion of a $\operatorname{symbol} a(x, \xi ; \mu) \in \widetilde{\mathbf{S}}_{1,0}^{d, v}$ takes the form

$$
a=\sum_{j=0}^{N-1} a_{[v+j]}^{\infty} \# \lambda^{d-v-j} \bmod \widetilde{S}_{1,0}^{d, v+N}
$$

note that here the Leibniz product actually coincides with the point-wise product of the involved symbols.

Definition 7.6 A family of order-reducing symbols is a set $\Lambda=\left\{\lambda^{\alpha}(x, \xi ; \mu) \mid \alpha \in \mathbb{R}\right\}$ of symbols $\lambda^{\alpha} \in S^{\alpha}$ which satisfy

(1) $\lambda^{0}=1 \bmod S^{-1}$,

(2) $\lambda^{\alpha} \# \lambda^{\beta}=\lambda^{\alpha+\beta} \bmod S^{\alpha+\beta-1}$ for every $\alpha, \beta \in \mathbb{R}$.

Note that any $\lambda^{\alpha}$ in such a family is parameter-elliptic in $S^{\alpha}$ and thus has a parametrix in $S^{-\alpha}$; this parametrix coincides with $\lambda^{-\alpha}$ modulo $S^{-\alpha-1}$.

Theorem 7.7 Let $\Lambda$ be a family of order-reducing symbols as in Definition 7.6. Then for a symbol $a(x, \xi ; \mu) \in \widetilde{S}_{1,0}^{d, v}$ the following are equivalent:

a) $a \in \widetilde{\mathbf{S}}_{1,0}^{d, \nu}$ (cf. Definition 5.1).

b) There exist $a_{[v+j]}^{\Lambda, \infty}(x, \xi) \in S_{1,0}^{\nu+j}\left(\mathbb{R}^{n}\right)$ such that, for every $N \in \mathbb{N}$,

$$
a=\sum_{j=0}^{N-1} a_{[v+j]}^{\Lambda, \infty} \# \lambda^{d-v-j} \bmod \widetilde{S}_{1,0}^{d, v+N}
$$

If $a_{[v]}^{\infty}(x, \xi)$ is the principal limit symbol of a then

$$
a_{[v]}^{\Lambda, \infty}=a_{[v]}^{\infty} \# \lambda_{0}^{-(d-v)}(x, 0,1),
$$

where $\lambda_{0}^{\alpha}(x, \xi ; \mu)$ denotes the homogeneous principal symbol of $\lambda^{\alpha}$. 
Before coming to the proof, let us show that the coefficients in any expansion of Theorem 7.7.b) are uniquely determined: Suppose $a=0$ and that we already have verified that $a_{[v+j]}^{\Lambda, \infty}=0$ for $j=0, \ldots, N-1$. Then $a_{[v+N]}^{\Lambda, \infty} \# \lambda^{d-v-N} \in \widetilde{S}_{1,0}^{d, v+N+1}$. Composing from the right with $\lambda^{-(d-v-N)}$ one finds

$$
a_{[v+N]}^{\Lambda, \infty}(x, \xi)=\left(a_{[v+N]}^{\Lambda, \infty} \# r_{0}\right)(x, \xi ; \mu)+r_{1}(x, \xi ; \mu)
$$

with some $r_{0} \in S^{-1}$ and $r_{1} \in \widetilde{S}_{1,0}^{v+N, v+N+1}$. The right-hand side decays as $1 / \mu$ in any semi-norm of $S_{1,0}^{\nu+N+1}\left(\mathbb{R}^{n}\right)$. Thus $a_{[v+N]}^{\Lambda, \infty}=0$.

Proof of Theorem 7.7 First we argue that we may assume without loss of generality that $v=0$. To this end let $p_{s}(\xi):=\langle\xi\rangle^{s}, s \in \mathbb{R}$. Then $p_{-v} \# a \in \widetilde{S}_{1,0}^{d^{\prime}, 0}$ for $d^{\prime}=d-v$.

Given hypothesis a), then $p_{-v} \# a \in \widetilde{\mathbf{S}}_{1,0}^{d^{\prime}, 0}$ and we show the existence of an expansion

$$
p_{-\nu} \# a=\sum_{j=0}^{N-1} b_{[j]}^{\Lambda, \infty} \# \lambda^{d^{\prime}-j} \bmod \widetilde{S}_{1,0}^{d^{\prime}, N} .
$$

Multiplying from the left with $p_{v}$ we find the desired expansion for $a$ with $a_{[v+j]}^{\Lambda, \infty}:=$ $p_{\nu} \# b_{[j]}^{\Lambda, \infty}$. We argue similarly when starting out from hypothesis $\mathrm{b}$ ).

Now let $v=0$; we show that b) implies a). By Proposition $5.3, \lambda^{d-j} \in \widetilde{\mathbf{S}}_{1,0}^{d-j, 0}$ has an expansion

$$
\lambda^{d-j}=\sum_{\ell=0}^{N-1} b_{j,[\ell]}^{\infty} \# \lambda^{d-j-\ell} \bmod \widetilde{S}_{1,0}^{d-j, N} ;
$$

in particular, $b_{j,[0]}^{\infty}(x)=\lambda^{d-j}(x, 0 ; 1)$. Therefore,

$$
\sum_{j=0}^{N-1} a_{[j]}^{\Lambda, \infty} \# \lambda^{d-j-\ell}=\sum_{j=0}^{N-1} \sum_{\ell=0}^{N-1} a_{[j]}^{\Lambda, \infty} \# b_{j,[\ell]}^{\infty} \# \lambda^{d-j-\ell} \quad \bmod \widetilde{S}_{1,0}^{d, N},
$$

since $a_{[j]}^{\Lambda, \infty} \# \widetilde{S}_{1,0}^{d-j, N} \subset \widetilde{S}_{1,0}^{d, N+j} \subset \widetilde{S}_{1,0}^{d, N}$ for every $j$. If $m:=j+\ell \geq N$,

$$
a_{[j]}^{\Lambda, \infty} \# b_{j,[\ell]}^{\infty} \# \lambda^{d-j-\ell} \in S_{1,0}^{m}\left(\mathbb{R}^{n}\right) \# S^{d-m} \subset \widetilde{S}_{1,0}^{m, m} \# \widetilde{S}_{1,0}^{d-m, 0} \subset \widetilde{S}_{1,0}^{d, m} \subset \widetilde{S}_{1,0}^{d, N} .
$$

We thus find

$$
a=\sum_{k=0}^{N-1} a_{[k]}^{\infty} \# \lambda^{d-k} \quad \bmod \widetilde{S}_{1,0}^{d, N}, \quad a_{[k]}^{\infty}:=\sum_{j+\ell=k} a_{[j]}^{\Lambda, \infty} \# b_{j,[\ell]}^{\infty} \in S_{1,0}^{k}\left(\mathbb{R}^{n}\right) .
$$

In particular, $a_{[0]}^{\infty}=a_{[0]}^{\Lambda, \infty} \# b_{0,[0]}^{\infty}=a_{[0]}^{\Lambda, \infty} \# \lambda_{0}^{d}(x, 0 ; 1)$.

Next we show that a) implies b) (again with $v=0$ ). We start out from the expansion

$$
a=\sum_{j=0}^{N-1} a_{[j]}^{\infty, 0} \# \lambda^{d-j} \bmod \widetilde{S}_{1,0}^{d, N} ;
$$

the additional super-script 0 is introduced for systematic reasons, since we will now establish an iterative procedure to transform this expansion in an expansion using the family $\Lambda$. Write

$$
a_{[0]}^{\infty, 0} \# \lambda^{d}=a_{[0]}^{\infty, 0} \#\left(\lambda^{d} \# \lambda^{-d}\right) \# \lambda^{d}+a_{[0]}^{\infty, 0} \# r_{0}
$$


with $r_{0} \in S^{d-1}$. By Proposition 5.3 we have expansions

$$
\begin{aligned}
\lambda^{d} \# \lambda^{-d} & =\sum_{j=0}^{N-1} b_{[j]}^{\infty} \# \lambda^{-j} \bmod \widetilde{S}_{1,0}^{0, N}, \\
r_{0} & =\sum_{j=0}^{N-2} r_{[j]}^{\infty} \# \lambda^{d-1-j} \bmod \widetilde{S}_{1,0}^{d-1, N-1} \subset \widetilde{S}_{1,0}^{d, N} .
\end{aligned}
$$

Inserting this in expansion (7.1) and using for $j \geq 1$ expansions

$$
\lambda^{-j} \# \lambda^{d}=\sum_{\ell=0}^{N-2} c_{j,[\ell]}^{\infty} \# \lambda^{d-j-\ell} \quad \bmod \widetilde{S}_{1,0}^{d-j, N-1} \subset \widetilde{S}_{1,0}^{d, N-1+j} \subset \widetilde{S}_{1,0}^{d, N},
$$

we find

$$
\begin{aligned}
\sum_{j=0}^{N-1} a_{[j]}^{\infty, 0} \# \lambda^{d-j}= & a_{[0]}^{\infty, 0} \# b_{[0]}^{\infty} \# \lambda^{d}+\sum_{j=1}^{N-1} \sum_{\ell=0}^{N-2} a_{[0]}^{\infty, 0} \# b_{[j]}^{\infty} \# c_{j,[\ell]}^{\infty} \# \lambda^{d-j-\ell} \\
& +\sum_{j=0}^{N-2}\left(a_{[j+1]}^{\infty, 0}+a_{[0]}^{\infty, 0} \# r_{[j]}^{\infty}\right) \# \lambda^{d-1-j} \bmod \widetilde{S}_{1,0}^{d, N} .
\end{aligned}
$$

The second term on the right-hand side equals

$$
\begin{aligned}
& \sum_{j=0}^{N-2} \sum_{\ell=0}^{N-2} a_{[0]}^{\infty, 0} \# b_{[j+1]}^{\infty} \# c_{j+1,[\ell]}^{\infty} \# \lambda^{d-1-j-\ell} \\
& \quad=\sum_{k=0}^{N-2}\left(\sum_{j+\ell=k}^{N-2} a_{[0]}^{\infty, 0} \# b_{[j+1]}^{\infty} \# c_{j+1,[\ell]}^{\infty}\right) \# \lambda^{d-1-k} \bmod \widetilde{S}_{1,0}^{d, N} .
\end{aligned}
$$

We conclude that

$$
a=\sum_{j=0}^{N-1} a_{[j]}^{\infty, 0} \# \lambda^{d-j}=a_{[0]}^{\Lambda, \infty} \# \lambda^{d}+\sum_{j=0}^{N-2} a_{[j+1]}^{\infty, 1} \# \lambda^{d-1-j} \bmod \widetilde{S}_{1,0}^{d, N} .
$$

with $a_{[0]}^{\Lambda, \infty}=a_{[0]}^{\infty, 0} \# b_{[0]}^{\infty}$ and resulting symbols $a_{[j+1]}^{\infty, 1} \in S_{1,0}^{j+1}\left(\mathbb{R}^{n}\right)$. This finishes the first step of the procedure. In the second step we write

$$
a_{[1]}^{\infty, 1} \# \lambda^{d-1}=a_{[1]}^{\infty, 1} \#\left(\lambda^{d-1} \# \lambda^{-(d-1)}\right) \# \lambda^{d-1}+a_{[1]}^{\infty, 1} \# r_{1}
$$

with $r_{1} \in S^{d-2}$ and proceed as above to finally obtain

$$
\sum_{j=0}^{N-2} a_{[j+1]}^{\infty, 1} \# \lambda^{d-1-j}=a_{[1]}^{\Lambda, \infty} \# \lambda^{d-1}+\sum_{j=0}^{N-3} a_{[j+2]}^{\infty, 2} \# \lambda^{d-2-j} \bmod \widetilde{S}_{1,0}^{d, N} .
$$

with resulting $a_{[1]}^{\Lambda, \infty}$ and $a_{[j+2]}^{\infty, 2} \in S_{1,0}^{j+2}\left(\mathbb{R}^{n}\right)$, hence

$$
a=a_{[0]}^{\Lambda, \infty} \# \lambda^{d}+a_{[1]}^{\Lambda, \infty} \# \lambda^{d-1}+\sum_{j=0}^{N-3} a_{[j+2]}^{\infty, 2} \# \lambda^{d-2-j} \quad \bmod \widetilde{S}_{1,0}^{d, N} .
$$


We iterate this procedure until the $N$-th step which consists in writing

$$
\sum_{j=0}^{0} a_{[j+N-1]}^{\infty, N-1} \# \lambda^{d-(N-1)-j}=a_{[N-1]}^{\infty, N-1} \# \lambda^{d-(N-1)}=a_{[N-1]}^{\Lambda, \infty} \# \lambda^{d-(N-1)} \bmod \widetilde{S}_{1,0}^{d, N} .
$$

resulting in

$$
a=\sum_{j=0}^{N-1} a_{[j]}^{\Lambda, \infty} \# \lambda^{d-j} \bmod \widetilde{S}_{1,0}^{d, N}
$$

as claimed in b). The proof is complete.

The following lemma will be useful in discussing localizations of operator-families.

Lemma 7.8 Let $a(x, \xi ; \mu) \in \widetilde{\mathbf{S}}_{1,0}^{d, v}$ have the expansion

$$
a=\sum_{j=0}^{N-1} a_{[v+j]}^{\Lambda, \infty} \# \lambda^{d-v-j} \bmod \widetilde{S}_{1,0}^{d, N}
$$

with respect to some family of order-reducing symbols $\Lambda$. Let $K \subset \mathbb{R}^{n}$ be a compact set and assume that $a \# \phi=$ a for every function $\phi \in \mathscr{C}_{\mathrm{comp}}^{\infty}\left(\mathbb{R}^{n}\right)$ with $\phi \equiv 1$ in an open neighborhood of $K$. Then, for every such function $\phi$ and every $j \geq 0$,

$$
a_{[v+j]}^{\Lambda, \infty} \# \phi=a_{[v+j]}^{\Lambda, \infty} .
$$

The analogous result for left-multiplication with $\phi$ holds also true (and follows trivially from the uniqueness of the coefficient-symbols in the expansion).

Proof We proceed by induction. Since

$$
a-a_{[v]}^{\Lambda, \infty} \# \lambda^{d-v} \in \widetilde{S}_{1,0}^{d, v+1},
$$

multiplication from the right with $\phi$ yields

$$
a-a_{[v]}^{\Lambda, \infty} \# \phi \# \lambda^{d-v}+a_{[v]}^{\Lambda, \infty} \#\left[\lambda^{d-v}, \phi\right] \in \widetilde{S}_{1,0}^{d, v+1},
$$

where $[\cdot, \cdot]$ is the commutator (with respect to \#). Now the third term belongs to

$$
S^{v}\left(\mathbb{R}^{n}\right) \# S^{d-v-1} \subset \widetilde{S}_{1,0}^{\nu, v} \# \widetilde{S}_{1,0}^{d-\nu-1} \subset \widetilde{S}_{1,0}^{d-1, \nu} \subset \widetilde{S}_{1,0}^{d, \nu+1} .
$$

Therefore,

$$
a-\left(a_{[v]}^{\Lambda, \infty} \# \phi\right) \# \lambda^{d-v} \in \widetilde{S}_{1,0}^{d, \nu+1} .
$$

The uniqueness of the coefficients in the expansion then implies $a_{[\nu]}^{\Lambda, \infty} \# \phi=a_{[\nu]}^{\Lambda, \infty}$.

Now suppose that (7.2) holds for $j=0, \ldots, N-1$. Given a function $\phi$ choose $\phi_{0} \in$ $\mathscr{C}_{0}^{\infty}\left(\mathbb{R}^{n}\right)$ such that $\phi_{0} \equiv 1$ near $K$ and $\phi \equiv 1$ near the support of $\phi_{0}$. Then, by induction assumption, we have

$$
\begin{aligned}
a=a \# \phi= & \sum_{j=0}^{N-1} a_{[v+j]}^{\Lambda, \infty} \# \phi_{0} \# \lambda^{d-v-j} \# \phi \\
& +a_{[v+N]}^{\Lambda, \infty} \# \phi \# \lambda^{d-v-N}+a_{[v+N]}^{\Lambda, \infty} \#\left[\lambda^{d-v-N}, \phi\right] \bmod \widetilde{S}_{1,0}^{d, v+N+1} .
\end{aligned}
$$


As above, the last term is shown to be in $\widetilde{S}_{1,0}^{d, v+N+1}$. Moreover, $\phi_{0} \# \lambda^{d-v-j} \#(1-\phi)$ belongs to $S^{-\infty}$. Using again the induction hypotheses we derive

$$
a=\sum_{j=0}^{N-1} a_{[v+j]}^{\Lambda, \infty} \# \lambda^{d-v-j}+\left(a_{[v+N]}^{\Lambda, \infty} \# \phi\right) \# \lambda^{d-v-N} \bmod \widetilde{S}_{1,0}^{d, v+N+1} .
$$

Thus, by uniqueness of the coefficients, (7.2) holds $j=N$.

Now lets turn to the global situation of operators on the manifold $M$. Let us fix some Riemannian metric $g$ on $M$.

Definition 7.9 A family of order-reducing operators on $M$ is a set $\Lambda=\left\{\Lambda^{\alpha}(\mu) \mid \alpha \in \mathbb{R}\right\}$ where $\Lambda^{\alpha}(\mu) \in L^{\alpha}$ has homogeneous principal symbol

$$
\sigma^{\alpha}\left(\Lambda^{\alpha}\right)(v ; \mu)=\left(|v|^{2}+\mu^{2}\right)^{\alpha / 2}
$$

and $\Lambda^{0}=1\left(|v|\right.$ denotes the modulus of a co-vector $v \in T^{*} M$ with respect to $g$ ).

Theorem 7.10 Let $A(\mu) \in \widetilde{\mathbf{L}}_{1,0}^{d, v}$. Then there exists uniquely determined operators $A_{[v+j]}^{\infty} \in$ $L_{1,0}^{v}(M), j \in \mathbb{N}_{0}$, such that, for every $N \in \mathbb{N}$,

$$
A(\mu)=\sum_{j=1}^{N-1} A_{[v+j]}^{\infty} \Lambda^{d-v-j}(\mu) \bmod \widetilde{L}_{1,0}^{d, \nu+N} .
$$

The leading coefficient $A_{[\nu]}^{\infty}$ is called the limit-operator of $A(\mu)$.

Proof The proof of the uniqueness is analogous to the one given after Theorem 7.7. Therefore, we shall focus on the existence of the expansion.

Let $\Omega_{1}, \ldots, \Omega_{m}$ be a covering of $M$ such that any union $\Omega_{i} \cup \Omega_{j}$ is contained in a chart(domain) of $M$. Let $\phi_{i} \in \mathscr{C}_{\text {comp }}^{\infty}\left(\Omega_{i}\right), i=1, \ldots, M$, be a sub-ordinate partition of unity. Then $A(\mu)=\sum_{i, j} \phi_{i} A(\mu) \phi_{j}$. It suffices to show the existence of an expansion for each summand.

Thus we may assume from the beginning that there exist a chart $\kappa: \Omega \rightarrow U$ and two functions $\phi, \psi \in \mathscr{C}_{\text {comp }}^{\infty}(\Omega)$ such that $A(\mu)=\phi A(\mu) \psi$. Let $a(x, \xi ; \mu) \in \widetilde{\mathbf{S}}_{1,0}^{d, v}$ be the symbol of $\kappa_{*} A(\mu)$ and let $K$ be the union of the supports of $\phi \circ \kappa^{-1}$ and $\psi \circ \kappa^{-1}$, respectively. $K$ is a compact subset of $U$.

Let $V$ be an open neighborhood of $K$ with compact closure contained in $U$. Take $\theta \in$ $\mathscr{C}_{\text {comp }}^{\infty}(U)$ with $\theta \equiv 1$ on $V$ and let $\lambda^{\alpha}(x, \xi ; \mu) \in S^{\alpha}$ be the symbol of $\kappa_{*}\left((\theta \circ \kappa) \Lambda^{\alpha}(\mu)(\theta \circ \kappa)\right)$. Note that

$$
\lambda^{\alpha}(x, \xi ; \mu)=\theta^{2}(x)\left(|\xi|_{x}^{2}+\mu^{2}\right)^{\alpha / 2} \chi(\xi, \mu)+r^{\alpha}(x, \xi ; \mu),
$$

where $\chi$ is a zero-excision function and $r^{\alpha} \in S^{\alpha-1}$. Now define

$$
\tilde{\lambda}^{\alpha}(x, \xi ; \mu)=\left(\theta(x)|\xi|_{x}^{2}+(1-\theta)(x)|\xi|^{2}+\mu^{2}\right)^{\alpha / 2} \chi(\xi, \mu)+r^{\alpha}(x, \xi ; \mu) ;
$$

then $\tilde{\Lambda}=\left\{\tilde{\lambda}^{\alpha} \mid \alpha \in \mathbb{R}\right\}$ is a family of order-reducing symbols in the sense of Definition 7.6 and $\tilde{\lambda}^{\alpha}(x, \xi, \mu)=\lambda^{\alpha}(x, \xi, \mu)$ whenever $x \in V$.

By Theorem 7.7 we have an expansion $a \sim \sum_{j} a_{[v+j]}^{\infty} \# \widetilde{\lambda}^{d-v-j}$. If $\theta_{0} \in \mathscr{C}_{\text {comp }}^{\infty}(V)$ with $\theta_{0} \equiv 1$ near $K$ then, by Lemma 7.8, $\theta_{0} a_{[v+j]}^{\infty}=a_{[v+j]}^{\infty} \# \theta_{0}=a_{[v+j]}^{\infty}$. Thus, taking another 
$\theta_{1} \in \mathscr{C}_{\text {comp }}^{\infty}(V)$ with $\theta_{1} \equiv 1$ near the support of $\theta_{0}$, we find

$$
a=\sum_{j=0}^{N-1} a_{[v+j]}^{\infty} \# \theta_{0} \tilde{\lambda}^{d-v-j} \# \theta_{1} \quad \bmod \theta_{0} \widetilde{S}_{1,0}^{d, v+N} \# \theta_{1} .
$$

Since $\theta_{0} \tilde{\lambda}^{d-\nu-j}=\theta_{0} \lambda^{d-\nu-j}$ by construction, and applying the pull-back under $\kappa$, we find

$$
A(\mu)=\sum_{j=1}^{N-1} A_{[v+j]}^{\infty}\left(\theta_{0} \circ \kappa\right) \Lambda^{d-v-j}(\mu)\left(\theta_{1} \circ \kappa\right) \bmod \widetilde{L}_{1,0}^{d, v+N}
$$

with $A_{[v+j]}^{\infty}=\kappa^{*} a_{[v+j]}^{\infty}(x, D)$. Finally, note that $\left(\theta_{0} \circ \kappa\right) \Lambda^{d-v-j}(\mu)\left(1-\theta_{1}\right) \circ \kappa \in L^{-\infty}$ due to the disjoint supports of $\theta_{0}$ and $1-\theta_{1}$ and that $A_{[v+j]}^{\infty}\left(\theta_{0} \circ \kappa\right)=A_{[v+j]}^{\infty}$.

Example 7.11 If $A(\mu) \in L^{d}$ then $A(\mu) \in \widetilde{\mathbf{L}}^{d, 0}$ as well; its limit-operator is the operator of multiplication with the function $\sigma(A)(x, 0 ; 1)$ (the homogeneous principal symbol of $A(\mu)$ evaluated in $(\xi, \mu)=(0,1))$.

Theorem 7.12 The limit-operator behaves multiplicative under composition: If $A_{j}(\mu) \in$ $\widetilde{\mathbf{L}}_{1,0}^{d_{j}, v_{j}}$ have limit-operator $A_{j,\left[v_{j}\right]}^{\infty}$ then $A_{0}(\mu) A_{1}(\mu) \in \widetilde{\mathbf{L}}_{1,0}^{d_{0}+d_{1}, v_{0}+v_{1}}$ has the limit-operator $A_{0,\left[\nu_{0}\right]}^{\infty} A_{1,\left[v_{1}\right]}^{\infty}$.

Proof In a first step, let $A(\mu) \in \widetilde{\mathbf{L}}_{1,0}^{d, v}$ have limit-operator $A_{[v]}^{\infty}$. Then

$$
\left.A_{[v]}^{\infty}=\lim _{\mu \rightarrow+\infty} A(\mu) \Lambda^{\nu-d}(\mu) \quad \text { (convergence in } L_{1,0}^{\nu+1}(M)\right) .
$$

In fact, using the expansion with $N=1$,

$$
A(\mu) \Lambda^{\nu-d}(\mu)=A_{[v]}^{\infty}+A_{[v]}^{\infty} R(\mu) \bmod \widetilde{L}_{1,0}^{v, v+1}
$$

with an $R(\mu) \in L^{-1} \subset S_{1,0}^{-1}\left(\overline{\mathbb{R}}_{+}, L_{1,0}^{0}(M)\right)$. Then $\widetilde{L}_{1,0}^{v, \nu+1} \subset S_{1,0}^{-1}\left(\overline{\mathbb{R}}_{+}, L_{1,0}^{\nu+1}(M)\right)$ yields the claim. Also one sees that $A(\mu) \Lambda^{\nu-d}(\mu)$ is bounded as a function of $\mu$ with values in $L^{v}(M)$.

Since $A_{0}(\mu) A_{1}(\mu) \in \widetilde{\mathbf{L}}_{1,0}^{d_{0}+d_{1}, \nu_{0}+v_{1}}$, it suffices to show that $A_{0}(\mu) A_{1}(\mu) \Lambda^{\nu_{0}+v_{1}-d_{0}-d_{1}}(\mu)$ converges to $A_{0,\left[\nu_{0}\right]}^{\infty} A_{1,\left[\nu_{1}\right]}^{\infty}$ in $L_{1,0}^{m}(M)$ for some $m \geq v_{0}+v_{1}+1$. Reasoning as before, we see that

$$
\begin{aligned}
A_{0}(\mu) A_{1}(\mu) \Lambda^{\nu_{0}+v_{1}-d_{0}-d_{1}}(\mu) & \equiv A_{0}(\mu) A_{1}(\mu) \Lambda^{\nu_{1}-d_{1}}(\mu) \Lambda^{\nu_{0}-d_{0}}(\mu) \\
& \equiv A_{0}(\mu) \Lambda^{\nu_{0}-d_{0}}(\mu) \Lambda^{d_{0}-\nu_{0}}(\mu) A_{1}(\mu) \Lambda^{\nu_{1}-d_{1}}(\mu) \Lambda^{\nu_{0}-d_{0}}(\mu)
\end{aligned}
$$

modulo terms belonging to $S_{1,0}^{-1}\left(\overline{\mathbb{R}}_{+}, L_{1,0}^{\nu_{0}+v_{1}}(M)\right)$. It remains to show that

$$
\Lambda^{d_{0}-v_{0}}(\mu)\left[A_{1}(\mu) \Lambda^{\nu_{1}-d_{1}}\right] \Lambda^{\nu_{0}-d_{0}}(\mu) \stackrel{\mu \rightarrow+\infty}{\longrightarrow} A_{1,\left[\nu_{1}\right]}^{\infty}
$$

in $L_{1,0}^{m}(M)$ for some $m \geq v_{1}+1$. Using the analogue of (7.3) for $A_{1}(\mu)$ this is readily seen to be equivalent to

$$
\Lambda^{d_{0}-v_{0}}(\mu) A_{1,\left[\nu_{1}\right]}^{\infty} \Lambda^{\nu_{0}-d_{0}}(\mu) \stackrel{\mu \rightarrow+\infty}{\longrightarrow} A_{1,\left[\nu_{1}\right]}^{\infty} .
$$

However, from Lemma 6.1 it follows that $\mu^{-\alpha} \Lambda^{\alpha}(\mu)$ is bounded in $L_{1,0}^{\alpha_{+}}(M)$ and, for $\mu \rightarrow$ $+\infty$, converges to 1 in $L_{1,0}^{\alpha_{+}+1}(M)$ for every $\alpha$. Therefore, (7.4) holds true with convergence in $L_{1,0}^{\nu_{1}+\left|d_{0}-v_{0}\right|+1}(M)$. 


\subsection{Extension to vector-bundles}

Given smooth vector-bundles $E_{j}, j=0,1$, on $M$ of dimension $n_{0}$ and $n_{1}$, respectively, the above definitions and results extend in a straight-forward way to operator-families acting as maps $\Gamma\left(M, E_{0}\right) \rightarrow \Gamma\left(M, E_{1}\right)$ between the spaces of smooth sections of $E_{0}$ and $E_{1}$, respectively. The definition of the spaces $\widetilde{L}_{1,0}^{d, v}\left(E_{0}, E_{1}\right), \widetilde{L}^{d, v}\left(E_{0}, E_{1}\right), \widetilde{\mathbf{L}}_{1,0}^{d, v}\left(E_{0}, E_{1}\right)$, and $\widetilde{\mathbf{L}}^{d, v}\left(E_{0}, E_{1}\right)$ uses local trivializations of the vector-bundles and $\left(n_{1} \times n_{0}\right)$-matrices $a(x, \xi ; \mu)=\left(a_{j k}(x, \xi ; \mu)\right)$ where the symbols $a_{j k}$ are from the corresponding symbolclasses $\widetilde{S}_{1,0}^{d, v}$, etc. We leave the details to the reader.

As above, given a bundle $E$, a family of order-reducing operators is a set $\Lambda_{E}$ of operators $\Lambda_{E}^{\alpha}(\mu) \in L^{\alpha}(E, E), \alpha \in \mathbb{R}$, which have (scalar-valued) principal symbol $\lambda_{0}^{\alpha}(x, \xi ; \mu)=$ $\left(|\xi|_{x}^{2}+\mu^{2}\right)^{\alpha / 2}$ and such that $\Lambda_{E}^{0}(\mu)$ is the identity operator. Then we obtain:

Theorem 7.13 Let $A(\mu) \in \widetilde{\mathbf{L}}_{1,0}^{d, v}\left(E_{0}, E_{1}\right)$. Then there exists uniquely determined operators $A_{[v+j]}^{\infty} \in L_{1,0}^{v}\left(E_{0}, E_{1}\right), j \in \mathbb{N}_{0}$, such that, for every $N \in \mathbb{N}$,

$$
A(\mu)=\sum_{j=1}^{N-1} A_{[v+j]}^{\infty} \Lambda_{E_{0}}^{d-v-j}(\mu) \bmod \widetilde{L}_{1,0}^{d, v+N}\left(E_{0}, E_{1}\right) .
$$

The leading coefficient $A_{[\nu]}^{\infty}$ is called the limit-operator of $A(\mu)$; it behaves multiplicatively under composition.

\subsection{Symbolic structure and ellipticity in $\tilde{\mathrm{L}}^{d, v}\left(E_{0}, E_{1}\right)$}

With any $A(\mu) \in \widetilde{\mathbf{L}}^{d, v}\left(E_{0}, E_{1}\right)$ we associate:

(1) the homogeneous principal symbol

$$
\sigma(A) \in \widetilde{\mathbf{S}}_{h o m}^{d, v}\left(\left(T^{*} M \backslash 0\right) \times \overline{\mathbb{R}}_{+} ; E_{0}, E_{1}\right)
$$

(a homomorphism acting between the pull-backs to $\left(T^{*} M \backslash 0\right) \times \overline{\mathbb{R}}_{+}$of the bundles $E_{0}$ and $E_{1}$, respectively),

(2) the principal angular symbol

$$
\widehat{\sigma}(A) \in S_{\text {hom }}^{d, v}\left(T^{*} M \backslash 0 ; E_{0}, E_{1}\right)
$$

(a homomorphism acting between the pull-backs to $T^{*} M \backslash 0$ of the bundles $E_{0}$ and $E_{1}$, respectively).

(3) the principal limit-operator $A_{[v]}^{\infty} \in L^{v}\left(M ; E_{0}, E_{1}\right)$.

Recall the compatibility relation

$$
\widehat{\sigma}(A)=\sigma\left(A_{[v]}^{\infty}\right),
$$

i.e., the principal angular symbol coincides with the homogeneous principal symbol of the limit-operator.

Proposition 7.14 Let $A(\mu) \in \widetilde{\mathbf{L}}^{d, v}\left(E_{0}, E_{1}\right)$ and assume that both homogeneous principal symbol and principal angular symbol are invertible on their domains. Then there exists a (rough) parametrix $B(\mu) \in \widetilde{\mathbf{L}}^{-d,-v}\left(E_{1}, E_{0}\right)$, i.e.,

$$
A(\mu) B(\mu)-1 \in \widetilde{\mathbf{L}}^{0-\infty, 0-\infty}\left(E_{1}, E_{1}\right), \quad B(\mu) A(\mu)-1 \in \widetilde{\mathbf{L}}^{0-\infty, 0-\infty}\left(E_{0}, E_{0}\right) .
$$


This result follows from the fact that the invertibility of a homogeneous principal symbol belonging to $\widetilde{\mathbf{S}}_{h o m}^{d, v}\left(\left(T^{*} M \backslash 0\right) \times \overline{\mathbb{R}}_{+} ; E_{0}, E_{1}\right)$ together with the invertibility of its angular symbol implies that its inverse belongs to the class $\widetilde{\mathbf{S}}_{h o m}^{-d,-v}\left(\left(T^{*} M \backslash 0\right) \times \overline{\mathbb{R}}_{+} ; E_{1}, E_{0}\right)$, cf. the local situation mentioned after Definition 5.11.

Definition 7.15 We call $A(\mu) \in \widetilde{\mathbf{L}}^{d, v}\left(E_{0}, E_{1}\right)$ elliptic if its homogeneous principal symbol is invertible on its domain and its principal limit-operator is invertible as a map $H^{s}\left(M, E_{0}\right) \rightarrow$ $H^{s-v}\left(M, E_{1}\right)$ for some $s^{3}$.

Theorem 7.16 Let $A(\mu) \in \widetilde{\mathbf{L}}^{d, v}\left(E_{0}, E_{1}\right)$ be elliptic. Then there exists a parametrix $B(\mu) \in$ $\widetilde{\mathbf{L}}^{-d,-v}\left(E_{1}, E_{0}\right)$ and $a \mu_{0} \geq 0$ such that

$$
A(\mu)^{-1}=B(\mu), \quad \mu \geq \mu_{0} .
$$

Proof By Proposition 7.14, there exists a rough parametric $B_{0}(\mu) \in \widetilde{\mathbf{L}}^{-d,-v}\left(E_{1}, E_{0}\right)$ such that $A(\mu) B_{0}(\mu)=1-R_{0}(\mu)$ with $R_{0}(\mu) \in \widetilde{\mathbf{L}}^{0-\infty, 0-\infty}\left(E_{1}, E_{1}\right)$.

Now let $B_{1}(\mu):=B_{0}(\mu)+\left(A_{[v]}^{\infty}\right)^{-1} R_{0,[-\infty]}^{\infty} \Lambda^{-(d-v)}$. Then

$$
B_{1}(\mu)-B_{0}(\mu) \in \widetilde{\mathbf{L}}^{-d-\infty,-v-\infty}\left(E_{1}, E_{0}\right) ;
$$

hence $B_{1}(\mu)$ is also a rough parametrix of $A(\mu)$, i.e., $A(\mu) B_{1}(\mu)=1-R_{1}(\mu)$ with $R_{1}(\mu) \in$ $\widetilde{\mathbf{L}}^{0-\infty, 0-\infty}\left(E_{1}, E_{1}\right)$. Moreover, $R_{1}(\mu)$ has vanishing limit-operator, since

$$
R_{1,[-\infty]}^{\infty}=1-A_{[\nu]}^{\infty}\left(B_{0,[-\nu]}^{\infty}+\left(A_{[v]}^{\infty}\right)^{-1} R_{0,[-\infty]}^{\infty}\right)=1-\left(1-R_{0,[-\infty]}^{\infty}\right)-R_{0,[-\infty]}^{\infty}=0 .
$$

Then, arguing as in Proposition 5.8, there exists an $S_{1}(\mu) \in \widetilde{\mathbf{L}}^{0-\infty, 0-\infty}\left(E_{1}, E_{1}\right)$ with vanishing limit-operator such that $\left(1-R_{1}(\mu)\right)\left(1-S_{1}(\mu)\right)=0$ for sufficiently large $\mu$. Thus, $B(\mu):=B_{1}(\mu)\left(1-S_{1}(\mu)\right)$ is a parametrix which yields a right-inverse of $A(\mu)$ for large $\mu$. Since we can construct in the same way a left-inverse of $A(\mu)$ for large $\mu$, the claim follows.

\subsection{Operators with finite regularity number}

In analogy to Sect. 5.4 we introduce the class

$$
\mathbf{L}^{d, v}\left(E_{0}, E_{1}\right)=\widetilde{\mathbf{L}}^{d, v}\left(E_{0}, E_{1}\right)+L^{d}\left(E_{0}, E_{1}\right), \quad v \in \mathbb{Z} .
$$

If $v$ is positive, the homogeneous principal symbol extends to a bundle homomorphism on $\left(T^{*} M \times \overline{\mathbb{R}}_{+}\right) \backslash 0$ and ellipticity means invertibility of this extended symbol. Then Theorem 5.21 generalizes in the obvious way to the global setting.

\subsection{Resolvent trace expansion}

Let us return to the resolvent-trace expansion of Grubb-Seeley. Let $\Lambda=\left\{r e^{i \theta} \mid 0 \leq \theta \leq \Theta\right\}$ and let $A \in L^{m}(M ; E, E), m \in \mathbb{N}$, be a $\psi$ do such that $\lambda-\sigma(A)$ is invertible on $\left(T^{*} M \times \Lambda\right) \backslash 0$. Moreover, let $Q \in L^{\omega}(M ; E, E)$. Theorem 6.4 together with integration over $M$ yields the following:

\footnotetext{
$\overline{3 \text { or, equivalently, there exists a } \psi}$ do $L_{1,0}^{-v}\left(M ; E_{1}, E_{0}\right)$ which is the inverse.
} 
Theorem 7.17 With the above notation and assumptions and $\ell \in \mathbb{N}$ such that $\omega-m \ell<$ $-\operatorname{dim} M$, there exist numbers $c_{j}, c_{j}^{\prime}, c_{j}^{\prime \prime}, j \in \mathbb{N}_{0}$, such that

$$
\operatorname{Tr} Q(\lambda-A)^{-\ell} \sim \sum_{j=0}^{+\infty} c_{j} \lambda^{\frac{n+\omega-j}{m}-\ell}+\sum_{j=0}^{+\infty}\left(c_{j}^{\prime} \log \lambda+c_{j}^{\prime \prime}\right) \lambda^{-\ell-\frac{j}{m}},
$$

uniformly for $\lambda \in \Lambda$ with $|\lambda| \longrightarrow+\infty$. Moreover, $c_{j}^{\prime}=c_{j}^{\prime \prime}=0$ whenever $j$ is not an integer multiple of $m$.

\subsection{Pseudodifferential operators of Toeplitz type}

Let us conclude with an application to so-called $\psi$ do of Toeplitz type, cf. [14,15].

To this end, for $j=0,1$, let $E_{j}$ be a vector-bundle over $M$ and $P_{j} \in L^{0}\left(M ; E_{j}, E_{j}\right)$ be idempotent, i.e., $P_{j}^{2}=P_{j}$. The $P_{j}$ define closed subspaces

$$
H^{s}\left(M, E_{j} ; P_{j}\right):=P_{j}\left(H^{s}\left(M, E_{j}\right)\right) \subseteq H^{s}\left(M, E_{j}\right)
$$

in the scale of $L_{2}$-Sobolev spaces $H^{s}$. Given $A(\mu) \in \widetilde{\mathbf{L}}^{d, 0}\left(E_{0}, E_{1}\right)$, consider

$$
\mathbf{A}(\mu):=P_{1} A(\mu) P_{0} \in \widetilde{\mathbf{L}}^{d, 0}\left(E_{0}, E_{1}\right) .
$$

We are interested in the invertibility of

$$
\mathbf{A}(\mu): H^{s}\left(M, E_{0} ; P_{0}\right) \longrightarrow H^{s-d}\left(M, E_{1} ; P_{1}\right) .
$$

Consider $P_{j}$ as an element in $\widetilde{\mathbf{L}}^{0,0}\left(E_{j}, E_{j}\right)$. Since $P_{j}$ is idempotent, so is the homogeneous principal symbol $\sigma\left(P_{j}\right)$ as morphism of the pull-back of $E_{j}$ to $\left(T^{*} M \backslash 0\right) \times \overline{\mathbb{R}}_{+}$, hence defines a sub-bundle denoted by $E_{j}\left(P_{j}\right)$.

Theorem 7.18 Let notations be as above. Assume that

(i) $\sigma(\mathbf{A}): E_{0}\left(P_{0}\right) \rightarrow E_{1}\left(P_{1}\right)$ is invertible,

(ii) $P_{1} A_{[0]}^{\infty} P_{0}: H^{s}\left(M, E_{0} ; P_{0}\right) \rightarrow H^{s}\left(M, E_{1} ; P_{1}\right)$ is invertible for some $s$.

Then there exists a $B(\mu) \in \widetilde{\mathbf{L}}^{-d, 0}\left(E_{1}, E_{0}\right)$ such that, for $\mathbf{B}(\mu):=P_{0} B(\mu) P_{1}$,

$$
\mathbf{B}(\mu) \mathbf{A}(\mu)=P_{0}, \quad \mathbf{A}(\mu) \mathbf{B}(\mu)=P_{1}
$$

for sufficiently large values of $\mu$. In particular, map (7.8) is an isomorphism for every choice of $s$ and $\mu$ large.

Proof For $j=0,1$ let us choose $S_{j}(\mu) \in L^{j d-s}\left(E_{j}, E_{j}\right)$ which are invertible for every $\mu \geq 0$ and with $S_{j}(\mu)^{-1} \in L^{s-d j}\left(E_{j}, E_{j}\right)$. Then

$$
\begin{aligned}
P_{j}^{\prime}(\mu) & :=S_{j}(\mu)^{-1} P_{j} S_{j}(\mu) \in L^{0}\left(E_{j}, E_{j}\right), \\
A^{\prime}(\mu) & :=S_{1}(\mu)^{-1} A(\mu) S_{0}(\mu) \in \widetilde{\mathbf{L}}^{0,0}\left(E_{0}, E_{1}\right) .
\end{aligned}
$$

Note that the $P_{j}^{\prime}(\mu)$ are (parameter-dependent) idempotents.

If $\mathbf{A}^{\prime}(\mu)=P_{1}^{\prime}(\mu) A^{\prime}(\mu) P_{0}^{\prime}(\mu)$ has a parametrix $\mathbf{B}^{\prime}(\mu)=P_{0}^{\prime}(\mu) B^{\prime}(\mu) P_{1}^{\prime}(\mu)$ with $B^{\prime}(\mu) \in$ $\widetilde{\mathbf{L}}^{0,0}\left(E_{1}, E_{0}\right)$ (i.e., the analog of (7.9) is true), then

$$
B(\mu):=S_{0}(\mu) B^{\prime}(\mu) S_{1}^{-1}(\mu)
$$


yields the desired parametrix $\mathbf{B}(\mu)$. However, this result follows from the general theory of abstract pseudodifferential operators and associated Toeplitz operators developed in $[14,15]$. In fact, in the notation of [15, Section 3.1] let $\Lambda=\overline{\mathbb{R}}_{+}$, let

$$
G=\{g=(M, E) \mid E \text { vector-bundle over } M\}
$$

be the set of all admissible weights and let $H^{0}(g)=L^{2}(M, E)$ for $g=(M, E)$. Moreover, for $g_{0}=\left(M, E_{0}\right), g_{1}=\left(M, E_{1}\right)$, and $\mathfrak{g}=\left(g_{0}, g_{1}\right)$ let $L^{0}(\mathfrak{g})=\widetilde{\mathbf{L}}^{0,0}\left(M ; E_{0}, E_{1}\right)$ and

$$
L^{-\infty}(\mathfrak{g})=\left\{A(\mu) \in \widetilde{\mathbf{L}}^{0-\infty, 0-\infty}\left(M ; E_{0}, E_{1}\right) \mid A_{[0]}^{\infty}=0\right\} .
$$

Now we can apply in [15, Theorem 1, Section 3.2], noting that (i), (ii) give the required hypotheses.

As a particular case we can take $\mathbf{A}(\mu)=P_{1}\left(\mu^{d}-A\right) P_{0}$ with a $\psi$ do $A \in L^{d}(M ; E, E)$, $d \in \mathbb{N}$, and two idempotents $P_{0}, P_{1} \in L^{0}(M ; E, E)$. Note that $A(\mu)=\mu^{d}-A$ considered as an element of $\widetilde{\mathbf{L}}^{d, 0}$ has limit-operator $A_{[0]} \equiv 1$, hence condition ii) in Theorem 7.18 reduces to the requirement that $P_{1}: H^{s}\left(M, E ; P_{0}\right) \rightarrow H^{s}\left(M, E ; P_{1}\right)$ isomorphically for some $s$.

Data Availability Statement Data sharing is not applicable to this article as no data sets were generated or analyzed during the current study.

Open Access This article is licensed under a Creative Commons Attribution 4.0 International License, which permits use, sharing, adaptation, distribution and reproduction in any medium or format, as long as you give appropriate credit to the original author(s) and the source, provide a link to the Creative Commons licence, and indicate if changes were made. The images or other third party material in this article are included in the article's Creative Commons licence, unless indicated otherwise in a credit line to the material. If material is not included in the article's Creative Commons licence and your intended use is not permitted by statutory regulation or exceeds the permitted use, you will need to obtain permission directly from the copyright holder. To view a copy of this licence, visit http://creativecommons.org/licenses/by/4.0/.

\section{Appendix: Calculus for symbols with expansion at infinity}

Let us provide the detailed proofs of Theorems 5.6 and 5.7. They are based on the concept of oscillatory integrals in the spirit of [7], but extended to Frèchet space valued amplitude functions. For an account on this concept see [2].

Let $E$ be a Fréchet space whose topology is described by a system of semi-norms $p_{n}$, $n \in \mathbb{N}$. A smooth function $q=q(y, \eta): \mathbb{R}^{m} \times \mathbb{R}^{m} \rightarrow E$ is called an amplitude function with values in $E$, provided there exist sequences $\left(m_{n}\right)$ and $\left(\tau_{n}\right)$ such that

$$
p_{n}\left(D_{\eta}^{\gamma} D_{y}^{\delta} q(y, \eta)\right) \lesssim\langle y\rangle^{\tau_{n}}\langle\eta\rangle^{m_{n}}
$$

for all $n$ and for all orders of derivatives. The space of such amplitude functions is denoted by $\mathcal{A}\left(\mathbb{R}^{m} \times \mathbb{R}^{m}, E\right)$. We shall frequently make use of the following simple observation:

Lemma 8.1 Let $E_{0}, E_{1}$ and $E$ be Fréchet spaces and let $((\cdot, \cdot))$ be a bilinear continuous map from $E_{1} \times E_{0}$ to $E$. If $q_{j}(y, \eta)$ are amplitude functions with values in $E_{j}, j=0,1$, then $q(y, \eta):=\left(\left(q_{1}(y, \eta), q_{0}(y, \eta)\right)\right)$ is an amplitude function with values in $E$.

If $\chi(y, \eta)$ denotes a cut-off function with $\chi(0,0)=1$, the so-called oscillatory integral

$$
\text { Os }-\iint e^{-i y \eta} q(y, \eta) d y d \eta:=\lim _{\varepsilon \rightarrow 0} \iint_{\mathbb{R}^{n} \times \mathbb{R}^{n}} e^{-i y \eta} \chi(\varepsilon y, \varepsilon \eta) q(y, \eta) d y d \eta
$$


exists and is independent of the choice of $\chi$. Note that for a continuous, $E$-valued function $f$ with compact support, $\iint f(y, \eta) d y d \eta$ is the unique element $e \in E$ such that $\left\langle e^{\prime}, e\right\rangle=$ $\iint\left\langle e^{\prime}, f(y, \eta)\right\rangle d y d \eta$ for every functional $e^{\prime} \in E^{\prime}$. For simplicity of notation we shall simply write $\iint$ rather than Os $-\iint$.

Proposition 8.2 Let $a \in \widetilde{\mathbf{S}}_{1,0}^{d, v}$. Then

$$
q(y, \eta):=((x, \xi, \mu) \mapsto a(x+y, \xi+\eta ; \mu)), \quad y, \eta \in \mathbb{R}^{n},
$$

defines an amplitude function $q \in \mathcal{A}\left(\mathbb{R}^{n} \times \mathbb{R}^{n}, \widetilde{\mathbf{S}}_{1,0}^{d, \nu}\right)$. The principal-limit symbol is

$$
q_{[v]}(y, \eta)=\left((x, \xi) \mapsto a_{[v]}^{\infty}(x+y, \xi+\eta)\right), \quad y, \eta \in \mathbb{R}^{n},
$$

(it is an amplitude function with values in $S_{1,0}^{v}\left(\mathbb{R}^{n}\right)$ ).

Analogous results hold true for $q_{1}(\eta):=((x, \xi, \mu) \mapsto a(x, \xi+\eta ; \mu))$ and $q_{2}(y):=$ $((x, \xi, \mu) \mapsto a(x+y, \xi ; \mu))$.

Proof Step 1: Suppose first that $a \in \widetilde{S}_{1,0}^{d, v}$ only. We show that $q$ is an amplitude function with values in $\widetilde{S}_{1,0}^{d, v}$.

Recall that the topology of $\widetilde{S}_{1,0}^{d, v}$ is defined by the semi-norms

$$
p_{N}(a):=\max _{|\alpha|+|\beta|+j \leq N} \sup _{x, \xi, \mu}\left|D_{\xi}^{\alpha} D_{x}^{\beta} D_{\mu}^{j} a(x, \xi ; \mu)\right|\langle\xi\rangle^{|\alpha|-v}\langle\xi, \mu\rangle^{\nu-d+j} .
$$

If $|\alpha|+|\beta|+j \leq N$ and $\gamma, \delta \in \mathbb{N}_{0}^{n}$ are arbitrary, then

$$
\begin{aligned}
& \left|D_{\xi}^{\alpha} D_{x}^{\beta} D_{\mu}^{j} D_{\eta}^{\gamma} D_{y}^{\delta} a(x+y, \xi+\eta ; \mu)\right| \leq C_{\gamma, \delta, N}\langle\xi+\eta, \mu\rangle^{d-\nu-j}\langle\xi+\eta\rangle^{\nu-|\alpha|-|\gamma|} \\
& \quad \leq C_{\gamma, \delta, N}\langle\xi, \mu\rangle^{d-\nu-j}\langle\eta\rangle^{|d-\nu-j|}\langle\xi\rangle^{\nu-|\alpha|}\langle\eta\rangle^{|\nu-| \alpha||} \\
& \quad \leq C_{\gamma, \delta, N}\langle\xi, \mu\rangle^{d-\nu-j}\langle\xi\rangle^{\nu-|\alpha|}\langle\eta\rangle^{m_{N}}
\end{aligned}
$$

with $m_{N}=\max \{|d-v-j|+|v-| \alpha|||| \alpha \mid+j \leq N\}$. This shows

$$
p_{N}\left(D_{\eta}^{\gamma} D_{y}^{\delta} q(y, \eta)\right) \lesssim\langle\eta\rangle^{m_{N}} .
$$

Step 2: Suppose $a \in \widetilde{\mathbf{S}}_{1,0}^{d, v}$. Then

$$
\begin{aligned}
a(x+y, \xi+\eta ; \mu)= & \sum_{j=0}^{N-1} a_{[v+j]}^{\infty}(x+y, \xi+\eta)[\xi+\eta, \mu]^{d-v-j}+ \\
& +r_{a, N}(x+y, \xi+\eta ; \mu) .
\end{aligned}
$$

According to Step $1, r_{a, N}(x+y, \xi+\eta ; \mu)$ defines an amplitude function with values in $\widetilde{S}_{1,0}^{d, v+N}$. In the same way one sees that $a_{[v+j]}^{\infty}(x+y, \xi+\eta)$ defines an amplitude function with values in $S_{1,0}^{\nu+j}\left(\mathbb{R}^{n}\right)$. By Lemma 8.3, $[\xi+\eta, \mu]^{d-\nu-j}$ defines an amplitude with values in $S^{d-v-j}$ hence, due to Proposition 5.3, with values in $\widetilde{\mathbf{S}}_{1,0}^{d-v-j, 0}$. Thus we can write, for every $M$,

$$
[\xi+\eta ; \mu]^{d-\nu-j}=\sum_{\ell=0}^{M-1} p_{j,[d-v-j+\ell]}^{\infty}(\eta, \xi)[\xi, \mu]^{d-\nu-j-\ell}+r_{j, M}(\eta, \xi ; \mu),
$$


where $p_{j,[d-v-j+\ell]}^{\infty}(\eta, \xi)$ defines an amplitude function with values in $S_{1,0}^{d-v-j+\ell}\left(\mathbb{R}^{n}\right)$ and $r_{j, M}(\eta, \xi ; \mu)$ an amplitude function with values in $\tilde{S}^{d-\nu-j, M}$. Note that one has $p_{j,[d-v-j]}^{\infty}(\eta, \xi) \equiv 1$. Inserting these expansions above and re-arranging terms, we find an expansion

$$
a(x+y, \xi+\eta ; \mu)=\sum_{j=0}^{N-1} \widetilde{a}_{[v+j]}^{\infty}(y, \eta ; x, \xi)[\xi, \mu]^{d-v-j}+R_{a, N}(y, \eta ; x, \xi, \mu)
$$

where $\tilde{a}_{[v+j]}^{\infty}(y, \eta ; x, \xi)$ and $R_{a, N}(y, \eta ; x, \xi, \mu)$ define amplitude functions with values in $\tilde{S}_{1,0}^{d-v+j}\left(\mathbb{R}^{n}\right)$ and $\widetilde{S}_{1,0}^{d-v, N}$, respectively. Note that $\widetilde{a}_{[v]}^{\infty}(y, \eta ; x, \xi)=a_{[v]}^{\infty}(x+y, \xi+\eta)$. Altogether, this shows the claims for $q$.

$q_{1}$ and $q_{2}$ are handled in the same way.

Lemma 8.3 Let $a(\xi ; \mu) \in S^{d}$. Then

$$
q(\eta)=((\xi, \mu) \mapsto a(\xi+\eta ; \mu)), \quad \eta \in \mathbb{R}^{n},
$$

defines an amplitude function with values in $S^{d}$.

Proof By Taylor expansion,

$$
a(\xi+\eta ; \mu)=\sum_{|\alpha|=0}^{N-1} \frac{1}{\alpha !} \partial_{\xi}^{\alpha} a(\xi ; \mu) \eta^{\alpha}+r_{N}(\eta, \xi ; \mu)
$$

with

$$
r_{N}(\eta, \xi ; \mu)=N \sum_{|\sigma|=N} \frac{\eta^{\sigma}}{\sigma !} \int_{0}^{1}(1-\theta)^{N-1}\left(\partial_{\xi}^{\sigma} a\right)(\xi+\theta \eta ; \mu) d \theta .
$$

Denoting by $r_{N, \sigma}(\eta, \xi ; \mu)$ the integral term, we have

$$
\begin{aligned}
\left|D_{\eta}^{\gamma} D_{\xi}^{\alpha} D_{\mu}^{j} r_{N, \sigma}(\eta, \xi ; \mu)\right| & \lesssim \int_{0}^{1}\langle\xi+\theta \eta, \mu\rangle^{d-N-|\alpha|-|\gamma|} d \theta \\
& \lesssim\langle\xi, \mu\rangle^{d-N-|\alpha|}\langle\eta\rangle^{|d-N-| \alpha||} .
\end{aligned}
$$

We conclude that $r_{N}(\eta, \xi ; \mu)$ defines an amplitude function with values in $S_{1,0}^{d-N}$. Write

$$
\partial_{\xi}^{\alpha} a(\xi ; \mu)=\chi(\xi, \mu) \sum_{j=0}^{N-1-|\alpha|} \partial_{\xi}^{\alpha} a_{j}(\xi ; \mu)+s_{\alpha, N}(\xi ; \mu)
$$

with a zero-excision function $\chi, a_{j} \in S_{h o m}^{d-j}$, and $s_{\alpha, N} \in S_{1,0}^{d-N}$. We find that

$$
a(\xi+\eta ; \mu)=\chi(\xi, \mu) \sum_{|\alpha|+j=0}^{N-1} \frac{1}{\alpha !} \partial_{\xi}^{\alpha} a_{j}(\xi ; \mu) \eta^{\alpha}+R_{N}(\eta, \xi ; \mu)
$$

with an amplitude function $R_{N}(\eta, \xi ; \mu)$ taking values in $S_{1,0}^{d-N}$. Therefore, the homogeneous components

$$
\left[q(\eta)_{k}\right](\xi ; \mu)=\sum_{|\alpha|+j=k} \frac{1}{\alpha !} \partial_{\xi}^{\alpha} a_{j}(\xi ; \mu) \eta^{\alpha}, \quad k \in \mathbb{N}_{0}
$$


are obviously amplitude functions with values in $S_{h o m}^{d-k}$. According to the definition of the topology of $S^{d}$ this shows the claim.

Theorem 8.4 The Leibniz product induces continuous maps

$$
\left(a_{1}, a_{0}\right) \mapsto a_{1} \# a_{0}: \widetilde{\mathbf{S}}_{1,0}^{d_{1}, v_{1}} \times \widetilde{\mathbf{S}}_{1,0}^{d_{0}, v_{0}} \longrightarrow \widetilde{\mathbf{S}}_{1,0}^{d_{0}+d_{1}, v_{0}+v_{1}}
$$

and the limit-symbol behaves multiplicatively: $\left(a_{1} \# a_{0}\right)_{\left[v_{0}+v_{1}\right]}^{\infty}=a_{1,\left[\nu_{1}\right]}^{\infty} \# a_{0,\left[\nu_{0}\right]}^{\infty}$. Moreover, for every $N \in \mathbb{N}_{0}$,

$$
a_{1} \# a_{0} \equiv \sum_{|\alpha|=0}^{N-1} \frac{1}{\alpha !}\left(\partial_{\xi}^{\alpha} a_{1}\right)\left(D_{x}^{\alpha} a_{0}\right) \quad \bmod \widetilde{\mathbf{S}}_{1,0}^{d_{0}+d_{1}-N, \nu_{0}+v_{1}-N} .
$$

Proof Recall that

$$
\left(a_{1} \# a_{0}\right)(x, \xi ; \mu)=\iint e^{-i y \eta} \underbrace{a_{1}(x, \xi+\eta ; \mu) a_{0}(x+y, \xi ; \mu)}_{=: p(y, \eta ; x, \xi, \mu)} d y d \eta .
$$

By Proposition 8.2, $p$ is an amplitude function with values in $\widetilde{\mathbf{S}}_{1,0}^{d_{0}+d_{1}, v_{0}+v_{1}}$, hence the oscillatory integral converges in this space.

Since the map $a \mapsto a_{[v]}^{\infty}: \widetilde{\mathbf{S}}_{1,0}^{d, v} \rightarrow S_{1,0}^{\nu}$ is linear and continuous, we find that

$$
\begin{aligned}
\left(a_{1} \# a_{0}\right)_{\left[v_{0}+v_{1}\right]}^{\infty}(x, \xi) & =\iint e^{-i y \eta} p(y, \eta ; x, \xi ; \mu)_{\left[v_{0}+v_{1}\right]}^{\infty} d y d \eta \\
& =\iint e^{-i y \eta} a_{1,\left[\nu_{1}\right]}^{\infty}(x, \xi+\eta) a_{0,\left[v_{0}\right]}^{\infty}(x+y, \xi) d y d \eta \\
& =\left(a_{0,\left[v_{0}\right]}^{\infty} \# a_{1,\left[v_{1}\right]}^{\infty}\right)(x, \xi) .
\end{aligned}
$$

Concerning expansion (8.1) recall that the difference of $a_{1} \# a_{0}$ and the sum in (8.1) is given by

$$
\begin{aligned}
r_{N}(x, \xi ; \mu)= & N \sum_{|\sigma|=N} \int_{0}^{1} \frac{(1-\theta)^{N-1}}{\sigma !} \times \\
& \times \iint e^{-i y \eta} \partial_{\xi}^{\sigma} a_{1}(x, \xi+\theta \eta ; \mu) D_{x}^{\sigma} a_{0}(y+x, \xi ; \mu) d y d \eta d \theta .
\end{aligned}
$$

Similarly as before, one can show that the integrand in (8.2) is an amplitude function with values in $\widetilde{\mathbf{S}}_{1,0}^{d_{0}+d_{1}-N, v_{0}+v_{1}-N}$, depending continuously on $\theta$. This yields the claim.

The proof of Theorem 5.7 is analogous, using

$$
a^{(*)}(x, \xi ; \mu)=\iint e^{-i y \eta} \overline{a(x+y, \xi+\eta ; \mu)} d y d \eta,
$$

and a similar formula for the remainder in (5.2).

\section{References}

1. Boutet de Monvel, L.: Boundary problems for pseudo-differential operators. Acta Math. 126(1-2), 11-51 (1971)

2. Gil, J.B., Schulze, B.-W., Seiler, J.: Cone pseudodifferential operators in the edge symbolic calculus. Osaka J. Math. 37(1), 221-260 (2000) 
3. Grubb, G.: Functional Calculus of Pseudo-differential Boundary Problems, 2nd edn. Birkhäuser, Basel (1996)

4. Grubb, G.: A weakly polyhomogeneous calculus for pseudodifferential boundary problems. J. Funct. Anal. 184(1), 19-76 (2001)

5. Grubb, G.: Regularity in $L_{p}$ Sobolev spaces of solutions to fractional heat equations. J. Funct. Anal. 274(9), 2634-2660 (2018)

6. Grubb, G., Seeley, R.T.: Weakly parametric pseudodifferential operators and Atiyah-Patodi-Singer boundary problems. Invent. Math. 121(3), 481-529 (1995)

7. Kumano-go, H.: Pseudo-Differential operators. MIT Press, Cambridge (MA) (1981)

8. Savin, A., Sternin, B.: Boundary value problems on manifolds with fibered boundary. Math. Nachr. 278(11), 1297-1317 (2005)

9. Schrohe, E., Schulze, B.-W.: Boundary value problems in Boutet de Monvel's calculus for manifolds with conical singularities I. In M. Demuth, E. Schrohe, B.-W. Schulze, editors, Pseudo-Differential Operators and Mathematical Physics, Math. Topics, Vol. 5: Advances in Part. Diff. Equ., Akademie Verlag, Berlin, (1994)

10. Schulze, B.-W.: Pseudo-differential Operators on Manifolds with Singularities. Studies in Math. and its Appl. 24, North-Holland Publishing Co., (1991)

11. Schulze, B.-W.: Boundary value problems and singular pseudo-differential operators. Wiley, Chichester, Pure and Applied Mathematics (New York) (1998)

12. Schulze, B.-W.: An algebra of boundary value problems not requiring Shapiro-Lopatinskij conditions. J. Funct. Anal. 179(2), 374-408 (2001)

13. Schulze, B.-W., Shatalov, V., Sternin, B.: On general boundary value problems for elliptic equations. Sb. Math. 189(9-10), 1573-1586 (1998)

14. Seiler, J.: Ellipticity in pseudodifferential algebras of Toeplitz type. J. Funct. Anal. 263(5), 1408-1434 (2012)

15. Seiler, J.: Parameter-dependent pseudodifferential operators of Toeplitz type. Ann. Mat. Pura Appl. 194(1), 145-165 (2015)

Publisher's Note Springer Nature remains neutral with regard to jurisdictional claims in published maps and institutional affiliations. 